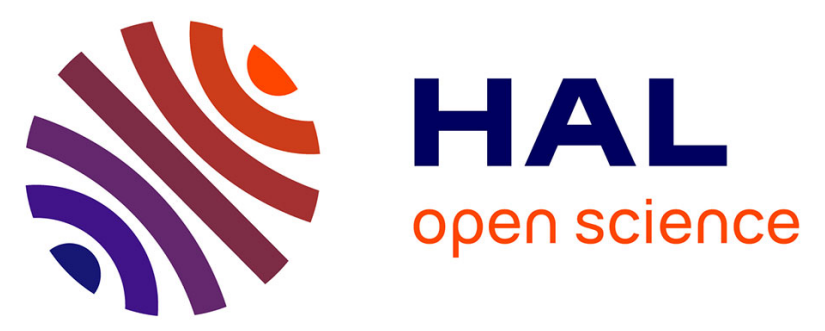

\title{
Three frontside full halo coronal mass ejections with a nontypical geomagnetic response
}

\author{
L. Rodriguez, A.N. Zhukov, C. Cid, Y. Cerrato, E. Saiz, H. Cremades, S. \\ Dasso, Michel Menvielle, A. Aran, C. Mandrini, et al.
}

\section{- To cite this version:}

L. Rodriguez, A.N. Zhukov, C. Cid, Y. Cerrato, E. Saiz, et al.. Three frontside full halo coronal mass ejections with a nontypical geomagnetic response. Space Weather: The International Journal of Research and Applications, 2009, 7 (6), pp.S06003. 10.1029/2008SW000453 . hal-00399242

\section{HAL Id: hal-00399242 \\ https://hal.science/hal-00399242}

Submitted on 7 Mar 2016

HAL is a multi-disciplinary open access archive for the deposit and dissemination of scientific research documents, whether they are published or not. The documents may come from teaching and research institutions in France or abroad, or from public or private research centers.
L'archive ouverte pluridisciplinaire HAL, est destinée au dépôt et à la diffusion de documents scientifiques de niveau recherche, publiés ou non, émanant des établissements d'enseignement et de recherche français ou étrangers, des laboratoires publics ou privés. 


\title{
Three frontside full halo coronal mass ejections with a nontypical geomagnetic response
}

\author{
L. Rodriguez, ${ }^{1}$ A. N. Zhukov, ${ }^{1,2}$ C. Cid, ${ }^{3}$ Y. Cerrato, ${ }^{3}$ E. Saiz, ${ }^{3}$ H. Cremades, ${ }^{4}$ \\ S. Dasso, ${ }^{5,6}$ M. Menvielle, ${ }^{7}$ A. Aran, ${ }^{8}$ C. Mandrini, ${ }^{5}$ S. Poedts, ${ }^{9}$ \\ and B. Schmieder ${ }^{10}$
}

Received 24 October 2008; revised 17 February 2009; accepted 17 March 2009; published 11 June 2009.

[1] Forecasting potential geoeffectiveness of solar disturbances (in particular, of frontside full halo coronal mass ejections) is important for various practical purposes, e.g., for satellite operations, radio communications, global positioning system applications, power grid, and pipeline maintenance. We analyze three frontside full halo coronal mass ejections (CMEs) that occurred in the year 2000 (close to the activity maximum of solar cycle 23), together with associated solar and heliospheric phenomena as well as their impact on the Earth's magnetosphere. Even though all three were fast full halos (with plane of the sky speeds higher than $1100 \mathrm{~km} / \mathrm{s}$ ), the geomagnetic response was very different for each case. After analyzing the source regions of these halo CMEs, it was found that the halo associated with the strongest geomagnetic disturbance was the one that initiated farther away from disk center (source region at W66); while the other two CMEs originated closer to the central meridian but had weaker geomagnetic responses. Therefore, these three events do not fit into the general statistical trends that relate the location of the solar source and the corresponding geoeffectivity. We investigate possible causes of such a behavior. Nonradial direction of eruption, passage of the Earth through a leg of an interplanetary flux rope, and strong compression at the eastern flank of a propagating interplanetary CME during its interaction with the ambient solar wind are found to be important factors that have a direct influence on the resulting north-south interplanetary magnetic field (IMF) component and thus on the CME geoeffectiveness. We also find indications that interaction of two CMEs could help in producing a long-lasting southward IMF component. Finally, we are able to explain successfully the geomagnetic response using plasma and magnetic field in situ measurements at the L1 point. We discuss the implications of our results for operational space weather forecasting and stress the difficulties of making accurate predictions with the current knowledge and tools at hand.

Citation: Rodriguez, L., et al. (2009), Three frontside full halo coronal mass ejections with a nontypical geomagnetic response, Space Weather, 7, S06003, doi:10.1029/2008SW000453.

\footnotetext{
${ }^{1}$ Solar-Terrestrial Center of Excellence, SIDC, Royal Observatory of Belgium, Brussels, Belgium.

${ }^{2}$ Skobeltsyn Institute of Nuclear Physics, Moscow State University, Moscow, Russia.

${ }^{3}$ Department of Physics, University of Alcala, Alcala de Henares, Spain.

${ }^{4}$ Facultad Regional Mendoza, Universidad Tecnológica Nacional, Mendoza, Argentina.
}

Copyright 2009 by the American Geophysical Union. 1542-7390/09/2008SW000453

\footnotetext{
${ }^{5}$ Instituto de Astronomía y Física del Espacio, CONICET, Universidad de Buenos Aires, Buenos Aires, Argentina.

${ }^{6}$ Departamento de Física, Facultad de Ciencias Exactas y Naturales, Universidad de Buenos Aires, Buenos Aires, Argentina.

${ }^{7}$ Centre d'Etude des Environnements Terrestre et Planétaires, Vélizy-Villacoublay, France.

${ }^{8}$ Department of Astronomy and Meteorology, University of Barcelona, Barcelona, Spain.

${ }^{9}$ Centre for Plasma Astrophysics, Catholic University Leuven, Leuven, Belgium.

${ }^{10}$ Observatoire de Paris, Meudon, France.
} 


\section{Introduction}

[2] Strong perturbations of the Earth's magnetosphere, such as geomagnetic storms, may have important effects on various activities of our technological society. The wide range of influence of geomagnetic field disturbances includes, for example, structuring the Earth's radiation belts (important for satellite operations), causing changes in the state of the ionosphere (influencing global positioning system applications and radio communications), creating geomagnetically induced currents (producing pipeline corrosion and power grid transformer saturation). Geomagnetic storm forecasting is thus an important problem for many practical applications [see, e.g., Bothmer and Daglis, 2007].

[3] Up to the early 1960s, the interplanetary causes of geomagnetic storms were not completely understood and were related normally to the plasma dynamics of the solar wind exclusively. Piddington [1963] suggested a possible relation between geomagnetic disturbances and the direction and strength of the interplanetary magnetic field (IMF). An important number of studies were published then in the 1960s and 1970s, where the southward component of the IMF was demonstrated to be the key interplanetary ingredient related to the production of geomagnetic storms [i.e., Rostoker and Fälthammar, 1967; Hirshberg and Colburn, 1969; Arnoldy, 1971; Meng et al., 1973; Russell et al., 1974; Burton et al., 1975; Patel and Wiskerchen, 1975; Perreault and Akasofu, 1978], which arise as a consequence of reconnection processes between the IMF and the magnetospheric field [Dungey, 1961]. An important issue in geomagnetic storm forecasting is then predicting the duration and magnitude of the southward IMF component on the base of solar observations.

[4] Coronal mass ejections (CMEs) and their interplanetary counterparts (ICMEs), which may contain long periods of strong southward IMF in the driver plasma itself and/or in the sheath plasma following the CMEdriven shock, are considered as the main sources of intense geomagnetic storms (see Tsurutani et al. [1988] and Gosling et al. [1990], see also reviews by Gonzalez et al. [1994, 1999], Schwenn [2006], and Bothmer and Zhukov [2007]). An important class of CMEs are halo CMEs [Howard et al., 1982], which are interpreted as CMEs propagating along the Sun-Earth line. Full halo CMEs have an apparent angular width of $360^{\circ}$, and, if coming from the front side of the Sun, give an early warning for a possible geomagnetic disturbance. Some papers have also reported interaction of solar and interplanetary structures as drivers of severe geomagnetic activity [Gonzalez et al., 1996; Y. M. Wang et al., 2003; Xie et al., 2006].

[5] When a frontside halo CME is detected in the field of view of a coronagraph placed on the Sun-Earth line, such as Solar and Heliospheric Observatory (SOHO)/ Large Angle and Spectrometric Coronagraph (LASCO) [Brueckner et al., 1995], the arrival of the corresponding ICME to the magnetosphere can be normally expected a few days later, with $t$ bsequent geomagnetic effect determined by the presence of southward IMF periods related to the transient. However, not every halo CME will arrive at the Earth. Schwenn et al. [2005] found that $15 \%$ of the 181 frontside halo CMEs they studied did not show any discernable signature in situ at the L1 point. In order to narrow down the possible arrivals, an investigation of the source region of the CME on the solar disk is normally undertaken by means of extreme ultraviolet (EUV), and $H \alpha$ observations. We use $\mathrm{SOHO} /$ Extreme Ultraviolet Imaging Telescope (EIT) data [Delaboudinière et al., 1995], which have a nearly continuous $24 \mathrm{~h}$ per day high-cadence coverage of the solar corona, as well as the Big Bear Solar Observatory $H \alpha$ data for this purpose. On-disk signatures like coronal dimmings (the most frequent one according to Delannee et al. [2000] and Zhukov $[2005,2007])$, EIT waves, posteruption arcades and erupting filaments allow the determination of the source region location and properties of erupting CMEs (e.g., their true angular width [see, e.g., Thompson et al., 2000; Zhukov and Veselovsky, 2007]).

[6] The longitudinal location of the source is of a vital importance. In general, events originating close to the limb and on the backside of the Sun have little chance of arriving at the Earth, whereas those occurring close to the Sun-Earth line have the highest. Furthermore, the events originating close to the limb, when they arrive at the magnetosphere, tend to be less geoeffective than those originating close to the central meridian and normally only a shock is observed [e.g., Manoharan et al., 2004; Zhukov, 2005] for fast CMEs, since the angular extent of the shock is larger than that of the corresponding CME. Akasofu and Fry [1986] found that (out of more than 200 cases) only one event originating at longitudes larger than $30^{\circ}$ from the solar central meridian resulted in a Dst $<-200 \mathrm{nT}$. In a recent study, Gopalswamy et al. [2007] analyzed 378 halo CMEs and found that halos originating at longitudes larger than $45^{\circ}$ from the central meridian were generally followed by moderate storms $(-50 \mathrm{nT}>D s t>$ $-100 \mathrm{nT})$, whereas halos originating closer to disk center (at longitudes less than $45^{\circ}$ from the central meridian) were found to be more strongly geoeffective (Dst $\leq-100 \mathrm{nT}$ ). This seems to be a consequence of the angular width of a typical CME around 50-60 [e.g., St. Cyr et al., 2000].

[7] In this work we present three cases of Sun-Earth connection events, which do not follow the general trends previously depicted. One of them originated close to the limb and produced a severe geomagnetic storm, while the other two started off close to the central meridian but generated a weak geomagnetic storm in one case and a moderate one in the other. The main goal of the paper is to present these nontypical cases in detail and analyze possible reasons of such an abnormal behavior, stressing the difficulties related to the real-time forecasting of geomagnetic activity with the current knowledge and tools at hand.

[8] The CMEs and their consequences will be described in the next section, where we also try to provide an 


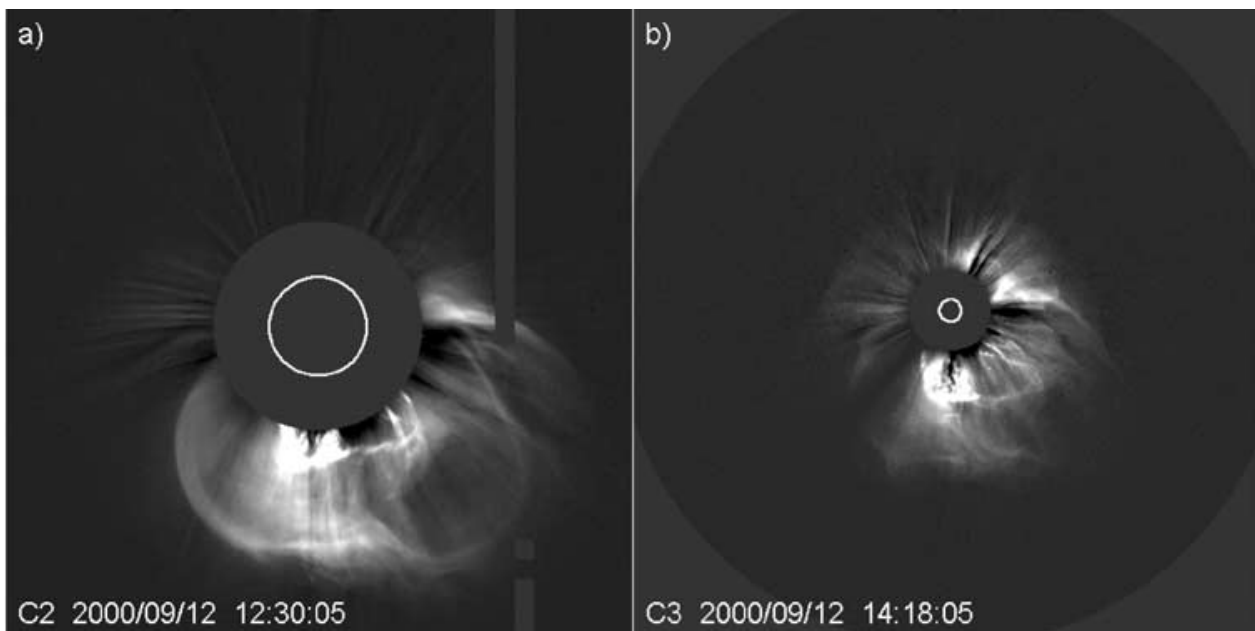

Figure 1. LASCO difference images of the halo CME on 12 September 2000. (a) LASCO C2, a preevent image at 1154:05 UT was subtracted. (b) LASCO C3, a preevent image at 1218:05 UT was subtracted.

explanation for their nontypical geomagnetic response based on solar and interplanetary observations. In section 3, we demonstrate that even for these nontypical cases the last step in the Sun-Earth connection (solar wind-magnetosphere interaction) can be well understood from the energetics point of view. Finally, in section 4, we will summarize and draw conclusions.

\section{Description of the Events}

\subsection{The 12 September 2000 Event}

[9] At 1206 UT a full halo CME appeared in the LASCO C2 field of view (see Figure 1). Its plane of the sky projected speed, according to the LASCO CME catalog [Yashiro et al., 2004], was $1550 \mathrm{~km} / \mathrm{s}$. This eruption could be tracked down to the low corona, where EIT observed an erupting filament and coronal dimmings, followed by a posteruption arcade (Figure 2). Associated with this event, an M1.0 flare peaking at 1213 UT was observed at S17W09. In $\mathrm{Ho}$ images, a filament was seen disappearing within the time interval of the event, shown in Figure 3. The filament had an approximated tilt angle of $60^{\circ}$ from the $\mathrm{N}-\mathrm{S}$ line. The orientations of its fibrils and barbs suggest a sinistral filament and right-handed chirality of the associated magnetic flux rope [see, e.g., Martin, 2003]. Using these solar observations as input, one could have forecasted the

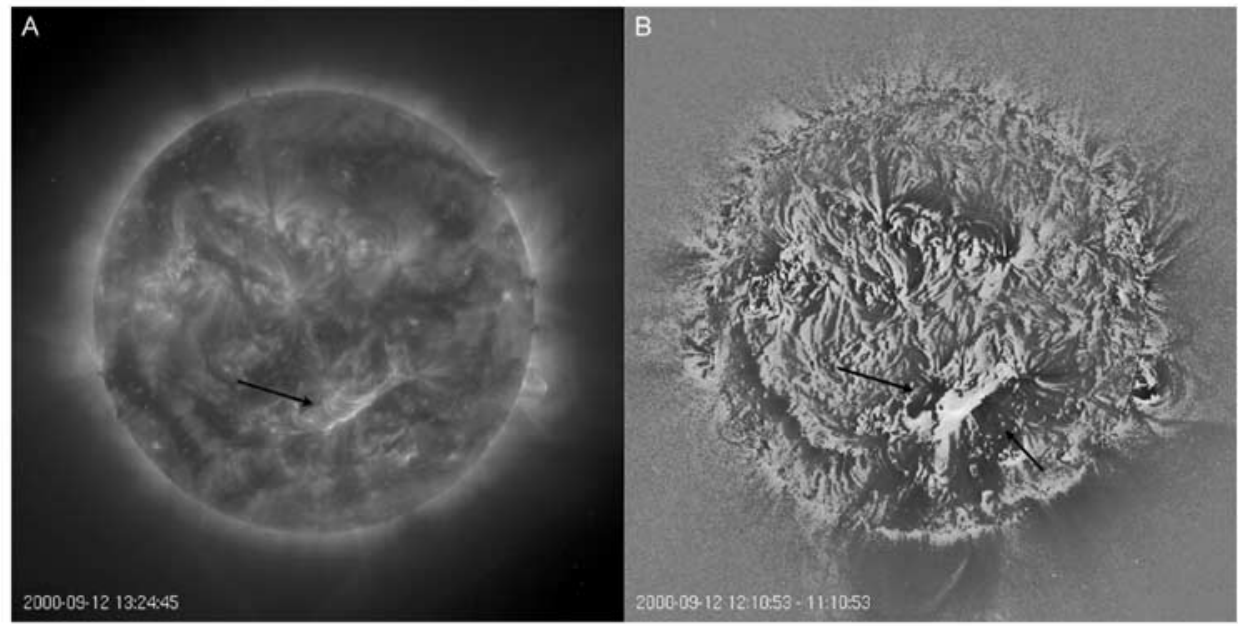

Figure 2. EIT images of the halo CME on 12 September 2000. The arrow in Figure 2a shows a posteruptive arcade, while those in Figure $2 b$ show the dimmings that were associated with the eruption. 


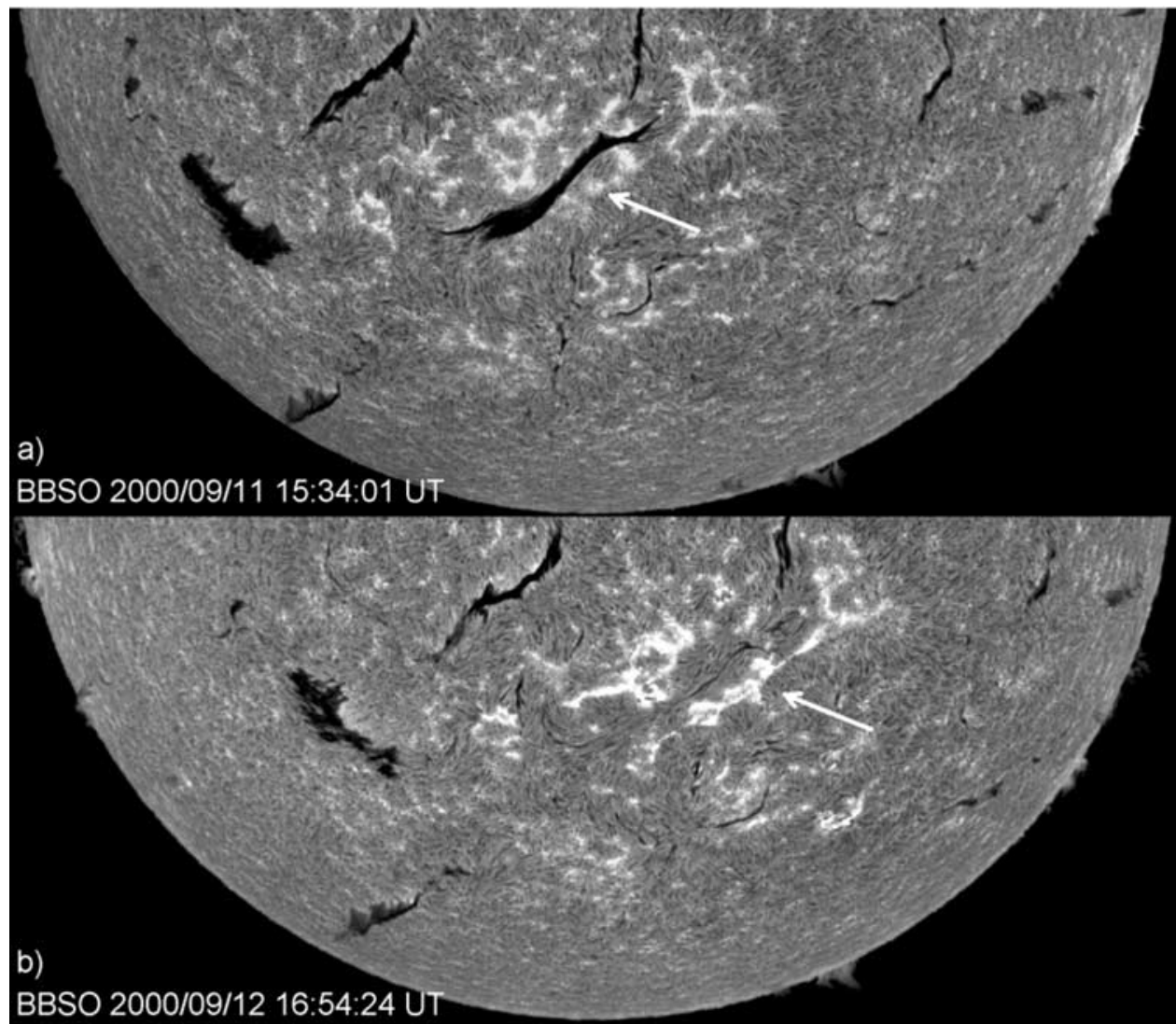

Figure 3. $\mathrm{H} \alpha$ images captured by the $20 \mathrm{~cm}$ refractor telescope at the Big Bear Solar Observatory (BBSO) on 12 September 2000. The arrows indicate the site of the disappearing filament associated with the CME.

arrival to the Earth of an ICME with the probable morphology of an interplanetary flux rope (magnetic cloud [see, e.g., Burlaga, 1991]), preceded by an interplanetary shock (because of the high speed of the CME).

[10] Three days later, on 15 September, a shock was indeed observed at the L1 point by the Advanced Composition Explorer (ACE) spacecraft at 0359 UT. The postshock plasma was propagating at moderate speeds (around $400 \mathrm{~km} / \mathrm{s}$ ). Even though the source region of this CME was located close to the central meridian, the shock was not accompanied by a discernable ICME in the data, as all parameters seem rather typical for a quiescent solar wind after shock passage (see Figure 4). No usual ICME signatures (see, e.g., reviews by Schwenn [2006], Zurbuchen and Richardson [2006], and Bothmer and Zhukov [2007]) were detected: there is neither a magnetic field rotation, nor a depletion of proton temperature, plasma beta is always close to one and the charge states of heavy ions remain within normal levels. In summary, there is no indication of an ICME related to this shock. Because of the absence of the strong, long-lasting southward IMF component in the postshock solar wind flow, the geoeffectiveness of the CME was weak with Dst reaching $-39 \mathrm{nT}$ (see Figure 4k).

[11] A calculation of the direction of the shock normal using a minimum variance analysis (MVA [Sonnerup and Cahill, 1967]) was carried out, using the magnetic field data corresponding to the shock. The orientation of the shock normal with respect to the ecliptic plane $(\Theta)$ was found to be $71^{\circ}$, while the angle $\Phi$ of the projection of the shock normal on the ecliptic plane, measured counterclockwise from the Earth-Sun direction, was found to be $217^{\circ}$. According to these values, it is plausible that the ICME driving the shock has passed to the south of the ecliptic plane and thus has missed the Earth, with ACE detecting only a wing of the shock at the L1 point. The LASCO data seem to confirm this hypothesis; even if the CME was a full halo, the bulk of the CME material was clearly propagating southward (Figure 1).

[12] This example indicates that the observation of a frontside full halo CME with a source region close to the central meridian does not necessarily mean a central collision with a resulting ICME. The distribution of mass in the halo may give an important indication on the 


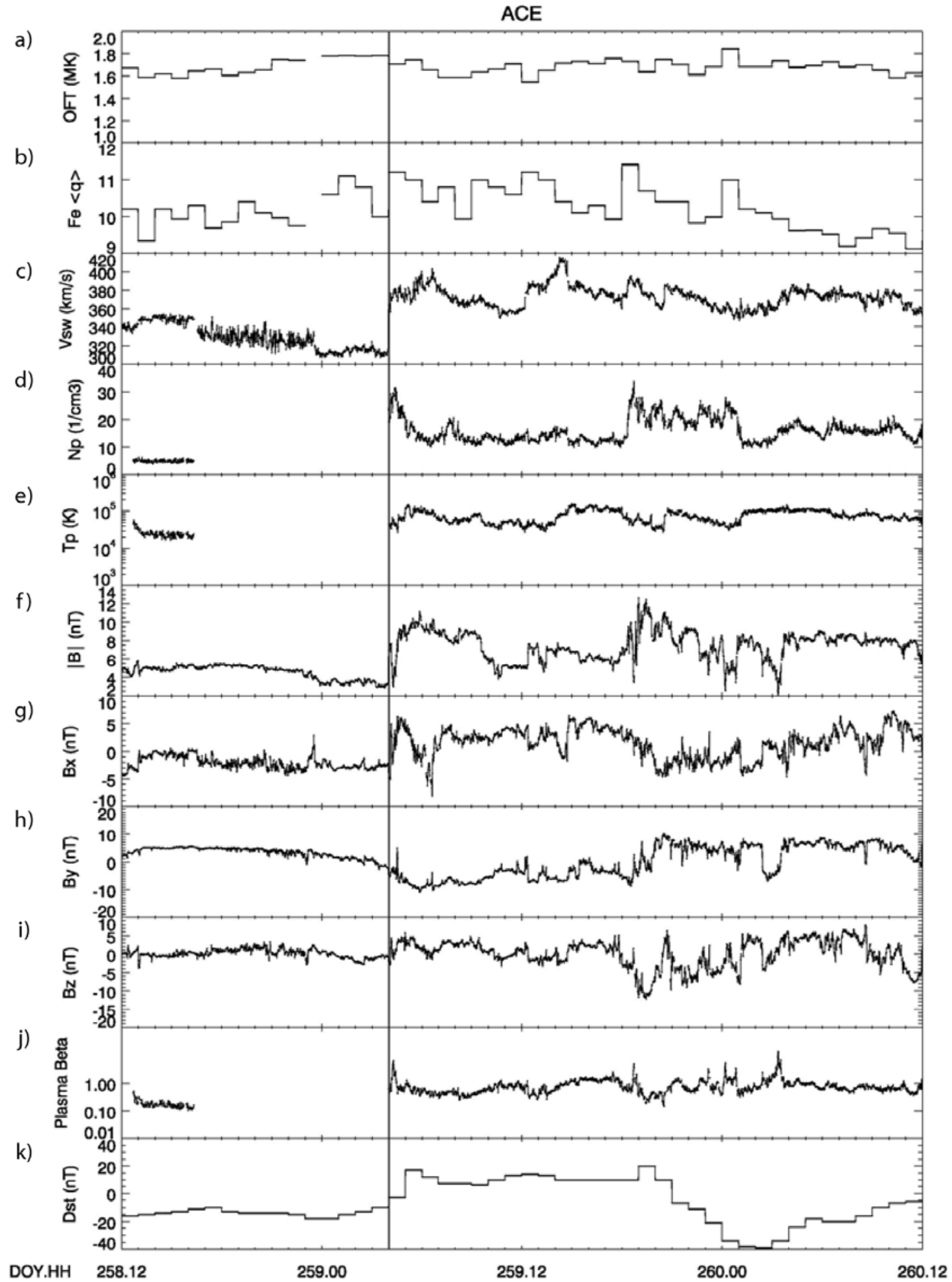

Figure 4 


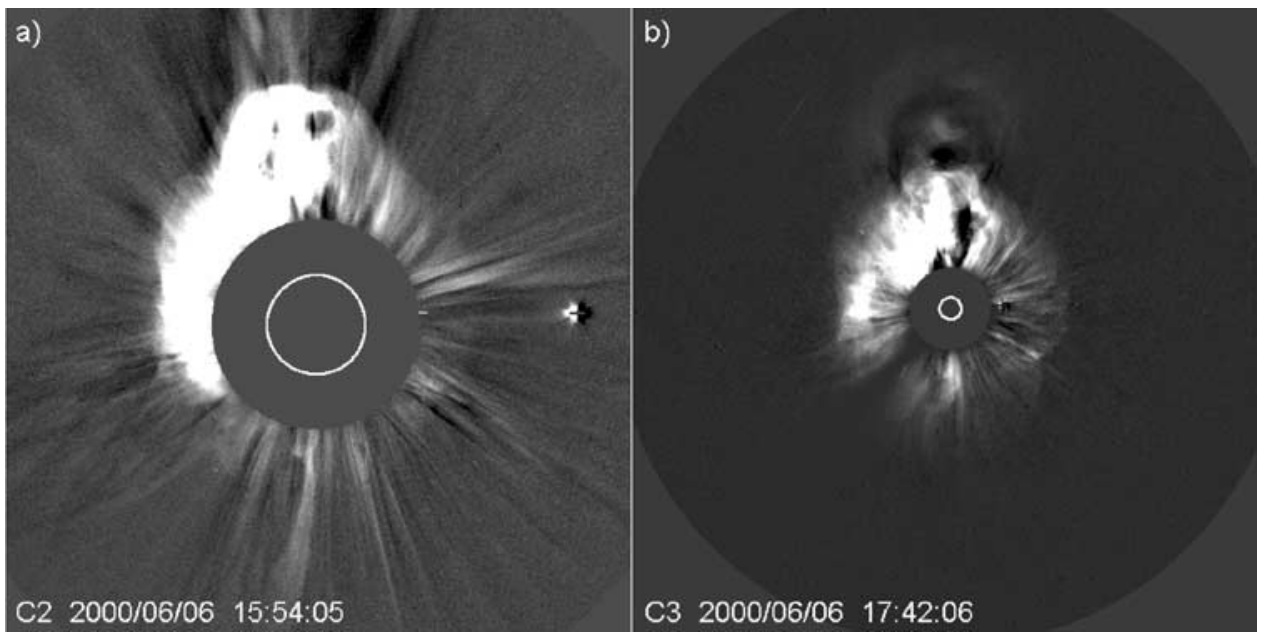

Figure 5. LASCO difference images of the halo CME on 6 June 2000. (a) LASCO C2, a preevent image at 1430:05 UT was subtracted. (b) LASCO C3, a preevent image at 1542:05 UT was subtracted.

direction of the eruption [see also Moon et al., 2005], which looks clearly nonradial close to the Sun.

\subsection{The 6 June 2000 Event}

[13] The first appearance of the CME in the LASCO C2 field of view was at 1554 UT (see Figure 5). LASCO difference images reveal that most of the CME material appears concentrated toward the NE, though the CME is classified as a full halo. According to the LASCO CME catalog, the projected speed on the plane of the sky was $1119 \mathrm{~km} / \mathrm{s}$, measured at a position angle of $\sim 45^{\circ}$ (counting counterclockwise from the North Pole). Coronal dimmings, EIT waves, and posteruptive loops were the CME signatures in the low corona detected by EIT as shown in Figure 6. Related to this event, an X2.3 flare peaking at 1458 UT was detected in NOAA AR 9026 at N20E18.

[14] In close temporal association with the halo CME, there was a north-directed CME seen first at 1530 UT by LASCO C2. A filament disappearance was detected in $\mathrm{H \alpha}$ (Figure 7) after 1421 UT (the cadence of the images available through the Global $H \alpha$ Network (http:// www.bbso.njit.edu/Research/Halpha/) precludes a more precise determination of the eruption time). According to the convention proposed by Martin [2003], the quiescent filament, as well as the accompanying fibrils, exhibit dextral chirality. In EIT images the filament is seen in absorption near the solar surface until 1524 UT. It is very difficult to unambiguously associate the filament with the first CME at 1530 UT or with the halo CME at 1554 UT. Temporal considerations, given that the filament is still present at $1524 \mathrm{UT}$, would put the halo CME as the most plausible candidate associated with the mentioned filament. However, the filament is seen rising northward, suggesting a spatial association with the CME starting at 1530 UT. In any case, both CMEs are launched within $\sim 25 \mathrm{~min}$, the first one with a speed about $200 \mathrm{~km} / \mathrm{s}$ lower than the second one, making an interaction possible after a few solar radii. From the point of view of a real-time forecaster, one can expect to observe at the Earth a shock followed by an ICME, possibly with a magnetic cloud morphology, although a more complicated configuration due to the CME interaction cannot be excluded.

[15] At $1 \mathrm{AU}, \mathrm{ACE}$ encountered a shock at $0841 \mathrm{UT}$ on 8 June. Shortly after, an ICME arrived at the L1 point, around 1600 UT on the same day. The plasma and magnetic field characteristics are shown in Figure 8. The signatures of this ICME are a low proton temperature and plasma beta and a strong magnetic field, accompanied by a smooth rotation of the magnetic field vector, together with a decreasing velocity profile (approximately from $800 \mathrm{~km} / \mathrm{s}$ to $600 \mathrm{~km} / \mathrm{s}$ ) showing a clear expansion of the structure. Because of the rotation in the magnetic field, this ICME can be classified as a magnetic cloud (MC). We fitted the cloud magnetic field with the flux rope model from Hidalgo et al. [2002] and obtained an orientation of the axis of $\Theta=11^{\circ}$ (elevation angle, measured with respect to the ecliptic

Figure 4. ACE data comprising the period that follows the arrival of the shock (vertical line) associated with the halo CME from 12 September 2000. No ICME can be found in the data. (a) Oxygen freezing-in temperature, (b) average iron charge state, (c) solar wind speed, (d) density, (e) temperature, (f) the magnetic field magnitude, $(\mathrm{g}-\mathrm{i})$ the components of the magnetic field vector (GSE system), (j) the proton plasma beta, and (k) the Dst data from World Data Center (WDC), Kyoto. Note that the missing data in $T p$ and $N p$ does not preclude us for finding the shock, we check also with Wind spacecraft and furthermore the shock is listed in the "ACE Lists of Disturbances and Tra ts" (http://www.bartol.udel.edu/ chuck/ace/ACElists/obs_list.html). 

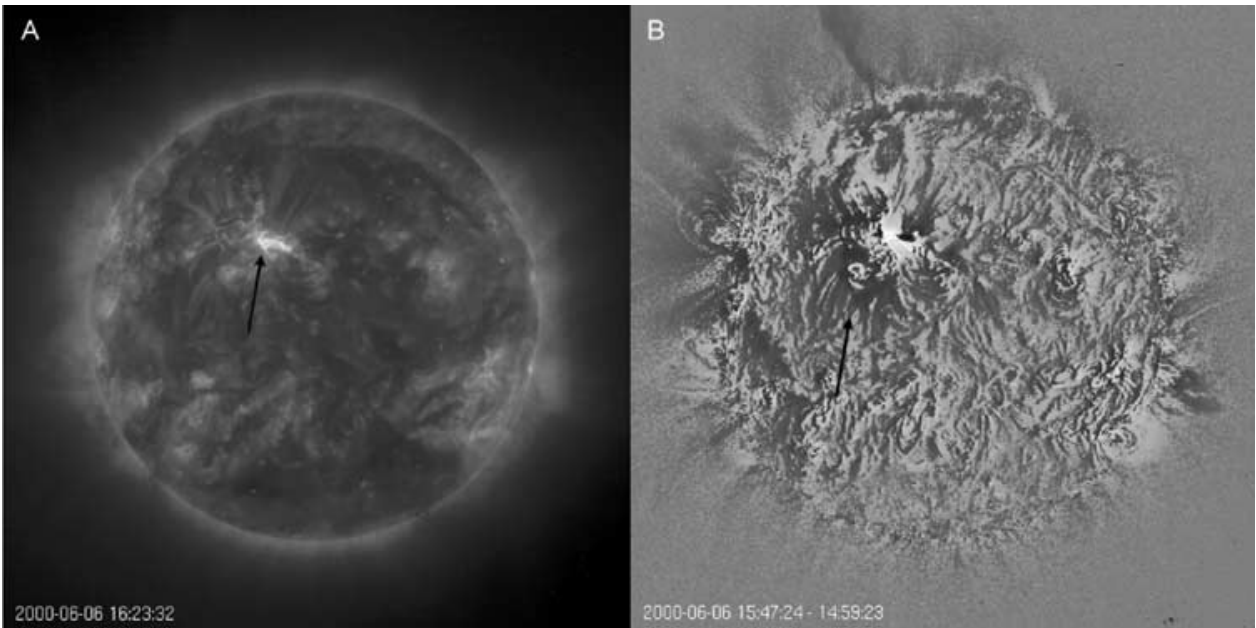

Figure 6. EIT images of the halo CME on 6 June 2000. The arrow in Figure 6a shows a posteruptive arcade, while that in Figure $6 \mathrm{~b}$ shows the dimmings that were associated with the eruption.

plane) and $\Phi=307^{\circ}$ (azimuthal angle, projection of the axis on the ecliptic plane, measured counterclockwise from the Earth-Sun direction), with a positive helicity (right-handed flux rope). The main contribution to the magnetic field intensity is provided by the Sun-Earth IMF component $B_{x}$. The behavior of this component suggests that ACE has passed through a leg of the MC. Since the erupting filament observed on the Sun (see previous paragraph) had a dextral chirality (corresponding to a left-handed flux rope), the MC observed at $1 \mathrm{AU}$, found to be right handed, can hardly be the interplanetary manifestation of this filament. Thus the filament was most likely related to the first CME and not to the halo CME.

[16] The trailing part of the MC was marked by an increase in the charge states of oxygen and iron which continued after the period we have marked as a MC, based mostly on the rotation of the magnetic field. According to
Richardson and Cane [2004], compositional anomalies might extend beyond the trailing part of the ejecta. Furthermore, the period marked as an MC could have been extended longer, considering that the plasma characteristics and the plasma beta remain at levels which could be encountered within ICMEs; but the magnetic field rotation ceases to be smooth after our chosen boundaries. A longer period for the ICME with respect to the rotation in the field components can be associated with the effect of reconnection in the front of the magnetic cloud, creating open field lines in its trailing part [Dasso et al., 2006].

[17] Coming back to the issue of the possible interaction between the two CMEs, we devoted special attention to the sheath region between the shock and the ICME. This region is marked by high density, temperature, magnetic field values and rapid variations in these parameters.

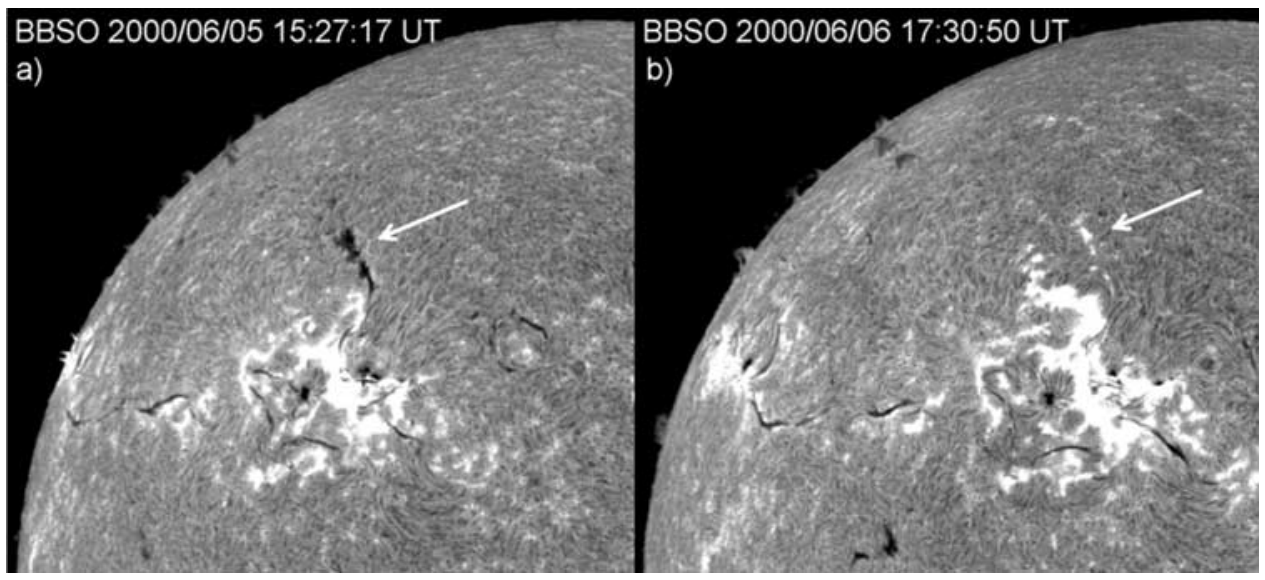

Figure 7. BBSO $\mathrm{H \alpha}$ images showing the disappearance of the filament associated with the CME ocurring on 62000 . 


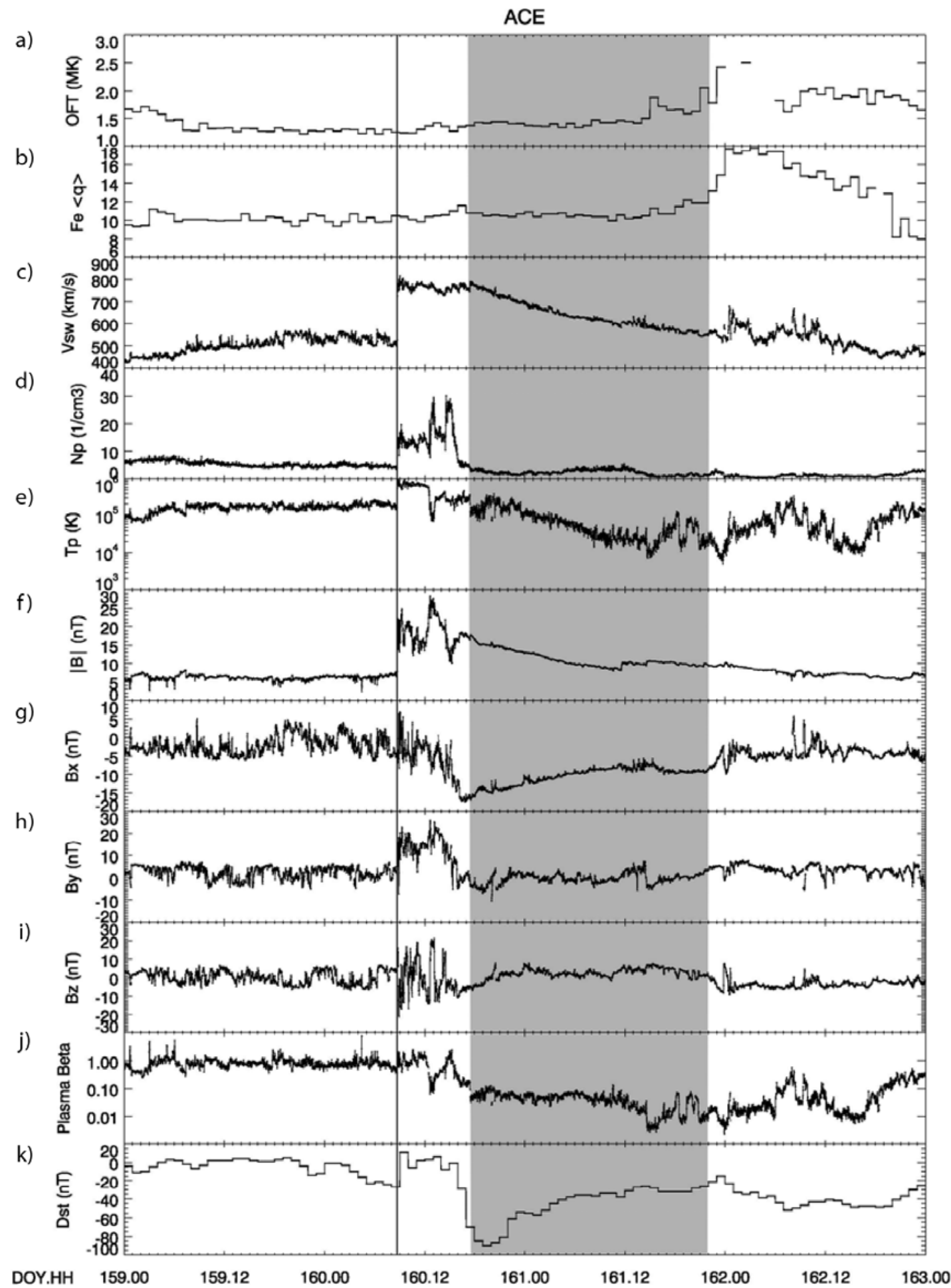

Figure 8. Magnetic field and plasma data obtained by ACE, for the period related to the halo CME from 6 June 2000. The arrival of the shock is marked by the vertical line. The shadowed area contains the ICME, which was a MC. (a) Oxygen freezing-in temperature, (b) average iron charge state, (c) solar wind speed, (d) density, (e) temperature, (f) the magnetic field magnitude, ( $\mathrm{g}-\mathrm{i})$ the components of the magnetic field vector (GSE system), (j) the proton plasma beta, and (k) the Dst data from WD yoto. 
Nevertheless, there is a brief period of time (between $\sim 12: 30$ and $\sim 1315$ UT) within the sheath where the temperature drops while the density and the magnetic field magnitude peak, producing low plasma beta values close to 0.1 (see zoomed version of the plasma and magnetic field data in Figure 9). These factors are not typical for the shocked plasma within a sheath region. They could be a remaining imprint of the first CME that was overtaken by the halo CME, close to the Sun.

[18] The fluctuations in $B_{z}$ before the MC reached relatively high values (down to $-16 \mathrm{nT}$ ), but the southward IMF orientation did not last for a considerable amount of time. Such behavior is characteristic for postshock sheath regions. Inside the magnetic cloud, the $B_{z}$ was only weakly negative. As a result, the geomagnetic response to this event was a moderate storm with Dst reaching $-90 \mathrm{nT}$ (Figure 8k). A stronger geomagnetic storm was not produced, despite the fact that it was a fast halo CME originating close to disk center.

[19] The lack of strong southward IMF inside the cloud can be explained by the geometry of the encounter. For a magnetic cloud with a low inclination to the ecliptic plane, one can expect to detect southward IMF either on the front or on the rear side of the cloud apex. However, the derived orientation of the MC axis $\left(\Theta=11^{\circ}\right.$ and $\left.\Phi=307^{\circ}\right)$ and the overall direction of the IMF inside the MC (mostly along the $x$ axis of the GSE coordinate system, i.e., along the Sun-Earth line) indicate that ACE was passing through a leg of the magnetic flux rope rather than through its apex.

[20] By looking at solar observations, a forecaster would not be able to reliably identify factors that would be responsible for a noncentral geometry of the MC collision with the Earth. Here again the interaction between the two CMEs may be playing a role.

\subsection{The 4 April 2000 Event}

[21] This full halo CME was first seen in the LASCO C2 field of view at $1632 \mathrm{UT}$, showing a brightness asymmetry toward the west (Figure 10). As suggested by Gopalswamy [2002], the faint structure surrounding the bulk of the ejection can be associated with the CME-driven shock observed in white light. The projected speed according to the LASCO CME catalog is $1188 \mathrm{~km} / \mathrm{s}$. Again, several typical signatures of an ejection appeared in EIT data: coronal dimmings (rapidly extending toward the south), an EIT wave and posteruptive loops (Figure 11). A C9.7 flare peaking at 1512 UT accompanied this event, located at N16W66. The disappearance of a long dextral filament with an almost vertical orientation (tilt angle with respect to the solar meridian around $12^{\circ}$ ), next to NOAA AR 8933, was observed in $\mathrm{H} \alpha$ (see Figure 12). Because of the position of the CME source region strongly toward the west limb, a real-time forecaster could have predicted that only the CME-driven shock would arrive at the Earth, followed by a sheath with rapidly fluctuating magnetic field. Because of expected IMF fluctuations with unknown amplitude inside the sheath, a moderate to intense resulting geomagnetic storm could have been expected.

[22] At ACE, a shock arrived on 6 April at 1604 UT, followed by a very long sheath region until 0900 UT on the following day (see Figure 13). The sheath is characterized by compressed magnetic field and plasma, showing high plasma temperature, density and magnetic field intensity, accompanied by strong fluctuations in the magnetic field components. Immediately after the sheath, an ICME was detected exhibiting a depletion in the plasma beta and in proton temperature. The ICME was expanding as it can be seen by looking at its velocity profile (decrease from around $640 \mathrm{~km} / \mathrm{s}$ to $550 \mathrm{~km} / \mathrm{s}$ ). We were unable to fit the ICME magnetic field with any flux rope model. The ICME is not a magnetic cloud. There was a weak flow of suprathermal bidirectional electrons detected by SWEPAM on ACE (not shown in Figure 13). Oxygen and iron charge states started to increase during the sheath region and continued at high values until the central part of the ICME. During the second half of the transient, the values returned to normal levels.

[23] The IMF $B_{z}$ component inside the ICME was weak and did not result in a significant geomagnetic activity. However, the interplanetary magnetic field inside the sheath had a strong (down to $-30 \mathrm{nT}$ ) long-lasting (up to $4 \mathrm{~h}$ ) $B_{z}$ component. During the ensuing geomagnetic storm the peak value of the Dst index reached $-288 \mathrm{nT}$, making this storm one of the seven largest geomagnetic storms seen in history of Dst variation [Jadav et al., 2005]. Such a high geoeffectiveness is surprising for an event originating near the west limb.

[24] In a detailed study of this event, Huttunen et al. [2002] estimated the shock normal orientation to be $\Theta=$ $-31^{\circ}$ and $\Phi=171^{\circ}$ (in solar ecliptic coordinates). As it was stated by those authors, the latitudinal angle $(\Theta)$ is consistent with the CME originating in the north hemisphere of the Sun, but the longitudinal one $(\Phi)$ seems too small considering the western origin of the disturbance. Furthermore, the presence of high-charge states early in the long sheath after the shock is not a common feature for the postshocked plasma. Normally a gradual increase can be seen starting in the rear part of sheath region and reaching a maximum value within the driver gas [Galvin et al., 1987; Richardson and Cane, 2004]. The early start of the highcharge states (translated in high freezing-in temperatures) is especially visible in iron that reached an average charge state value of 15 (equivalent to $\sim 2.510^{6} \mathrm{~K}$ ) shortly after the shock passage.

[25] In order to explain the two inconsistencies listed in the previous paragraph we have searched for activity on the Sun that could have affected the halo CME. The interaction of the ICME with a high-speed stream (noted by Xie et al. [2006]) possibly emanating from the faint coronal hole situated a few degrees eastward (seen in EIT data) might have affected the ICME. According to Gonzalez et al. [1996], CMEs occurring close to low-latitude coronal 


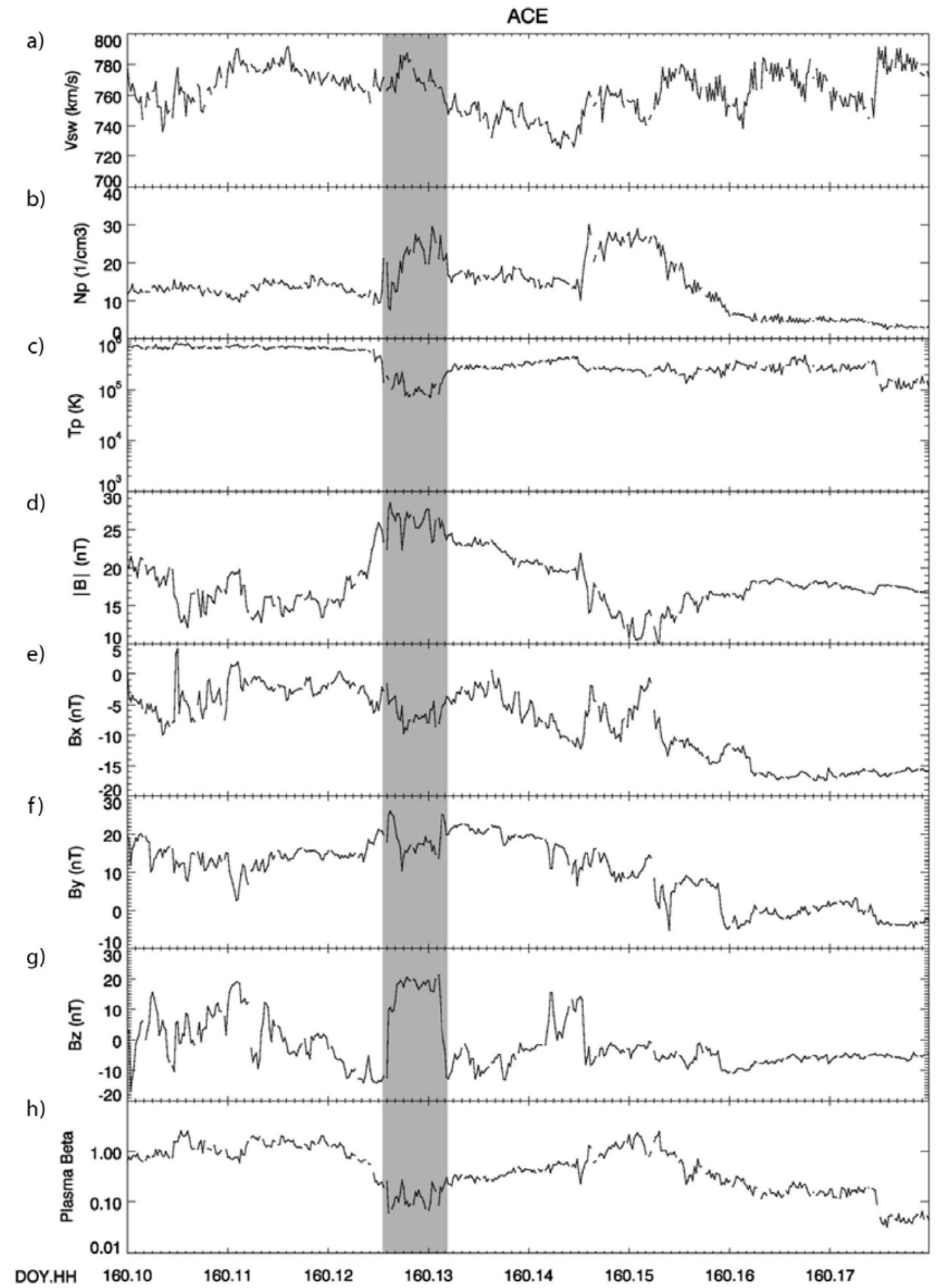

Figure 9. Zoomed version on the sheath region from the CME on 6 June 2000. The region of interest (see text for details) is marked by the shadowed area. (a) Solar wind speed, (b) density, (c) temperature, $(\mathrm{d})$ the magnetic field magnitude, $(\mathrm{e}-\mathrm{g})$ the components of the magnetic field vector (GSE s stem), and (h) the proton plasma beta. 


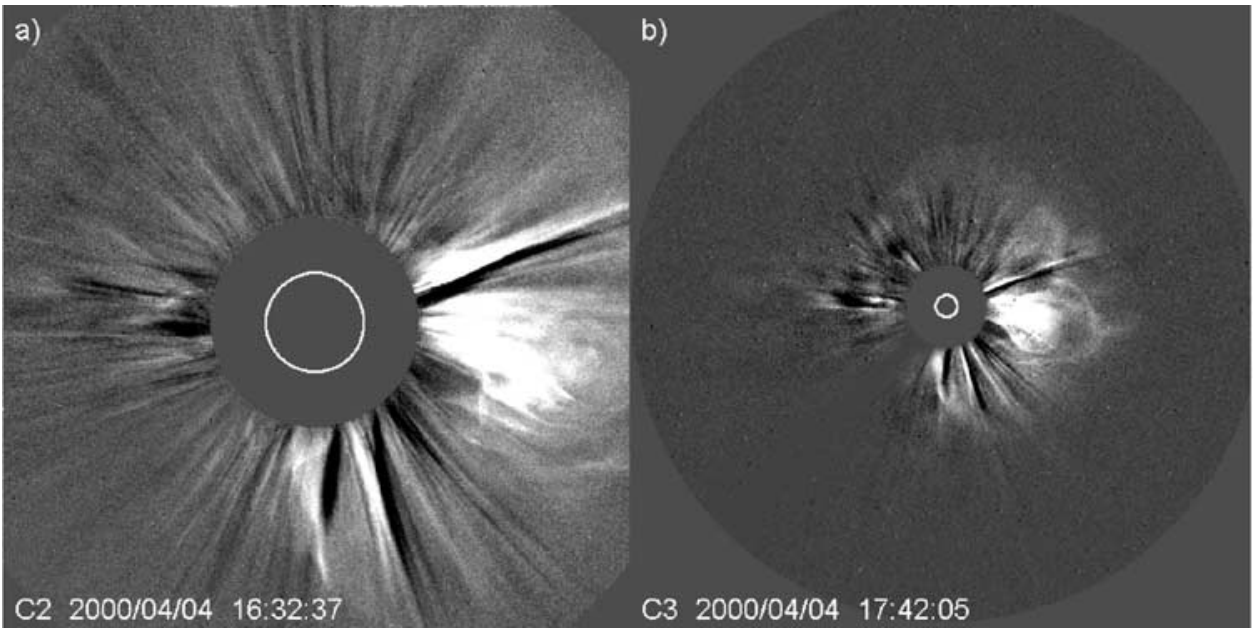

Figure 10. LASCO difference images of the halo CME on 4 April 2000. (a) LASCO C2, a preevent image at 1506:05 UT was subtracted. (b) LASCO C3, a preevent image at 1518:05 UT was subtracted.

holes may have an increased geoeffectivity. Nevertheless, it would not account for the elevated charge states appearing clearly before the main ICME material and early within the sheath. These charge states seem to be related with ICME material and not with the ambient coronal plasma which would be normally swept by the shock. Investigating the LASCO data in more detail, we have identified a weak partial halo CME first appearing in the C2 field of view at 0630 UT on the same day, 4 April. It is not listed as a halo in the CME catalog by Yashiro et al. [2004], but we measured its width to be around $170^{\circ}$ as it covers the position angle range from $180^{\circ}$ to $350^{\circ}$ (counting counterclockwise from the North Pole). This eruption can be tracked down to coronal dimmings in NOAA AR 8932, located at S12W46. This slow CME (398 km/s according to the LASCO catalog) has a high probability of being overtaken by the main event occurring several hours later. The interaction between the first, weaker and slower, CME with the strong event that came afterward could provide an explanation for the composition anomalies seen at $1 \mathrm{AU}$ in ACE data. The main CME encountered the second one in interplanetary space and then swept in the plasma of the first CME. This may explain why the charge states seen in the postshock region do not correspond to normal solar wind values (with freezing-in temperatures close to $1.510^{6} \mathrm{~K}$ ), but are more in agreement with the CME plasma. It is possible that the shock geometry was then also altered, leading to the small longitudinal angle as obtained by Huttunen et al. [2002]. Finally, the interaction between

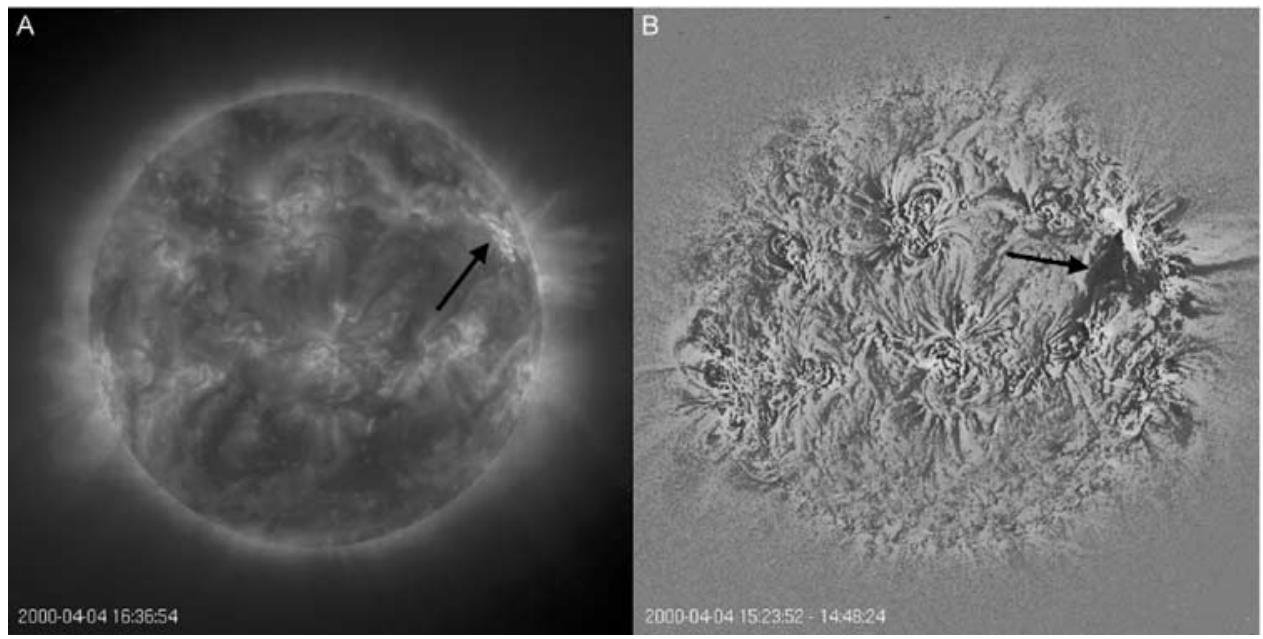

Figure 11. EIT images of the halo CME on 4 April 2000. The arrow in Figure 11a shows a posteruptive arcade, while that in Figure 11b shows the dimmings that were associated with the eruption. 


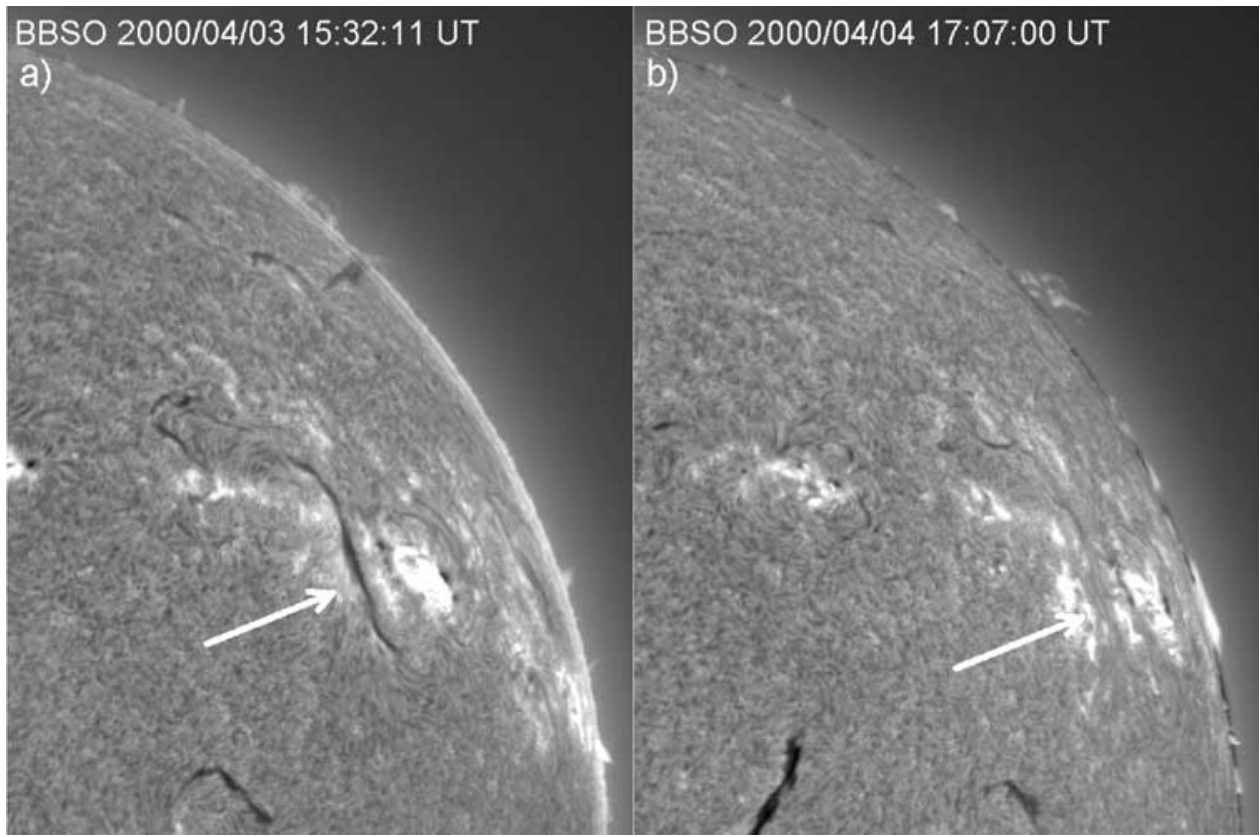

Figure 12. BBSO $\mathrm{H} \alpha$ images showing the disappearance of the filament associated with the CME occurring on 4 April 2000.

the two ICMEs (and possibly the coronal hole) may have contributed to the eventual geoeffectiveness of the disturbance.

[26] Another interesting possibility to produce highly compressed IMF in the sheath is the ambient magnetic field line draping about a fast ICME [see, e.g., Gosling and McComas, 1987]. This mechanism can lead to the appearance of the southward IMF during the whole duration of the sheath. This southward IMF can also be compressed by the CME-driven shock, thus increasing the $B_{z}$ strength. Siscoe et al. [2007] has demonstrated that because of the Parker spiral geometry of the ambient magnetic field, the region of the magnetic field compression is generally strongly shifted toward the east flank of the ICME sheath, thus increasing geoeffectiveness of CMEs originating in the western solar hemisphere. Finally, the eastward deflection of fast ICMEs due to the magnetic tension force from the spiral IMF in the slower ambient solar wind flow [Gosling et al., 1987] is another factor that can contribute to the arrival of the ICME plasma to the Earth from a western hemisphere event. It is possible that in this event all the mechanisms mentioned above have combined their effect and produced very strong values of the southward IMF. Unfortunately, the dependence of the amplitude of these effects on the observable solar parameters is still unclear.

\section{Explaining the Geoeffectiveness and the Dst Index From L1 Measurements}

[27] In order to measure the response of the terrestrial environment to solar are used [e.g., Menvielle and Berthelier, 1991]. Specifically, the Dst index measures the variations in the geomagnetic North-South component and it is constructed using the horizontal component of the geomagnetic field from four observatories at low to midlatitudes [Sugiura, 1965; Sugiura and Kamei, 1991]. The Dst index is considered a proxy for the deviation of the horizontal component of the geomagnetic field at the equator from a quiet day. Although it was introduced as a measurement of the ring current, other sources also contribute to this index such as the magnetopause and magnetotail currents, and induced currents in the solid Earth. The Dst index can be corrected to remove the contribution of these currents using the expression of Burton et al. [1975]

$$
D s t^{*}=D s t-b P^{1 / 2}+c,
$$

where $D s t^{*}$ is the magnetic field deviation caused by the ring current, $P$ is the solar wind dynamic pressure, and $b$ and $c$ are empirical coefficients. In this paper, when needed, we will use the correction from O'Brien and McPherron [2000], setting the values of these coefficients to $b=7.26$ and $c=11$, with solar wind dynamic pressure in $\mathrm{nPa}$ and Dst in $\mathrm{nT}$.

[28] A relationship of proportionality between the decrease of the horizontal component of the geomagnetic field and the energy content of the ring current was proposed by Dessler and Parker [1959] and Sckopke [1966]. As a result, $D s t^{*}$ can be considered as a proxy for the energy injected into the inner magnetosphere, which 


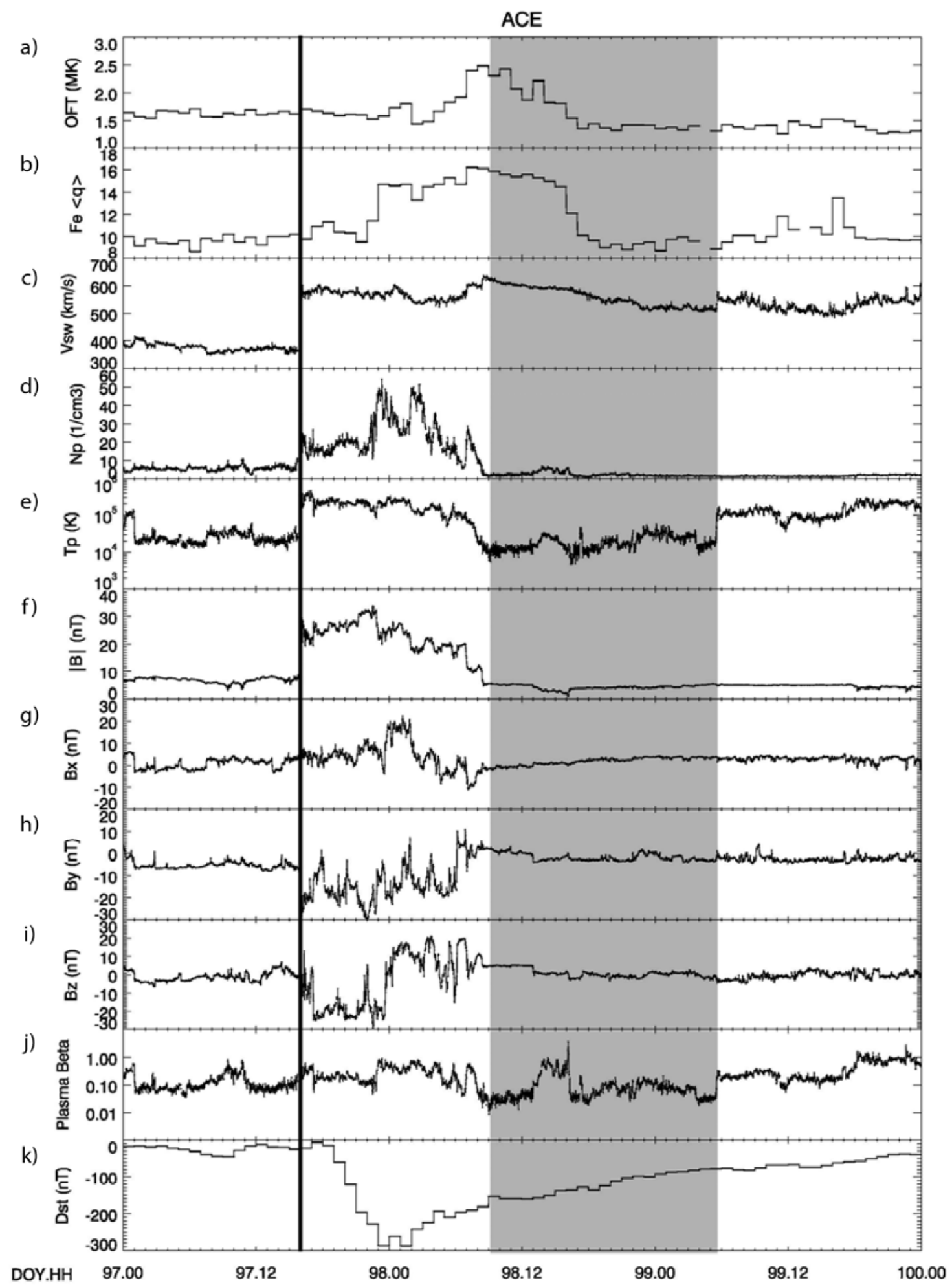

Figure 13. Magnetic field and plasma data obtained by ACE, for the period related to the halo CME from 4 April 2000. The arrival of the shock is marked by the vertical line. The shadowed area contains the ICME. (a) Oxygen freezing-in temperature, (b) average iron charge state, (c) solar wind speed, (d) density, (e) temperature, (f) the magnetic field magnitude, (g-i) the components of the magnetic field vector (G ystem), (j) the proton plasma beta, and (k) the Dst data from WDC, Kyoto. 
enhances the kinetic energy of the ring current population. In this scenario, and considering the energy balance for the ring current, it is possible to describe the evolution of $D s t^{*}$ by the following differential equation [Burton et al., 1975]

$$
\frac{d D s t^{*}(t)}{d t}=Q(t)-\frac{D s t^{*}(t)}{\tau},
$$

where $Q(t)$ corresponds to the injection function resulting from the interaction between solar wind and the terrestrial magnetosphere and energy losses (second term on the right-hand side of the equation) have been assumed proportional to the energy content of the ring current itself, being $\tau$ the decay time, with a typical value of $14 \pm 4 \mathrm{~h}$ for geomagnetic storms having a peak of Dst $<-100 \mathrm{nT}$ [Dasso et al., 2002].

[29] The injection function has not been related to solar observations, but several relationships have been obtained between the decrease in Dst* and solar wind parameters. The first one was proposed by Burton et al. [1975] from the statistical analysis of the solar wind data from years 1967 and 1968 measured on Explorer 33 and 35. They concluded that $D s t^{*}$ variations are proportional to the duskward interplanetary electric field (IEF). Then, the geoeffectiveness of a solar wind disturbance will depend on the intensity and duration of the duskward IEF that will develop as the disturbance travels away from the Sun [C. B. Wang et al., 2003].

[30] The evolution of $D s t^{*}$ is described as the balance between a source term and a decay one. As a consequence, the decrease rate of $D s t^{*}$ shall be related to the injection function, $Q(t)$ of equation (2), which represents the energy rate released from solar wind to terrestrial magnetosphere. Several injection functions, as well as several recovery times, have been proposed in the literature from empirical studies with the aim of providing an accurate theoretical forecast of the Dst index. For this study we use two of these $Q(t)$ functions: the one proposed by Burton et al. [1975], the first one proposed in literature, and that of $C$. B. Wang et al. [2003] (hereafter Burton and Wang injection functions, respectively).

[31] Concerning the Burton injection function, $Q(t)$ is treated as being uniquely determined by duskward interplanetary electric field or $V B_{s}$ (where $V$ is the solar wind speed and $B_{s}$ is the southward component of the magnetic field in GSM coordinates). Its functional dependence is given by

$$
Q_{B}(n T / h)=\left\{\begin{array}{cc}
-5.4(V B s-0.5) & V B s>0.5 \\
0 & V B s \geq 0.5
\end{array},\right.
$$

where $V B_{s}$ is expressed in $\mathrm{mV} / \mathrm{m}$. On the other hand, the solar wind pressure effect on the ring current is taken into account in the Wang injection function in the following way:

$$
Q_{W}(n T / h)=\left\{\begin{array}{cc}
-4.4(V B s-0.49)\left(\frac{P}{3.0}\right)^{0.2} & V B s>0.49 \\
0 & V B s \geq 0.49
\end{array},\right.
$$

with $V B_{s}$ in $\mathrm{mV} / \mathrm{m}$ and $P$ in $\mathrm{nPa}$.

[32] In Figure 14, the Burton and Wang injection functions and the $D s t^{*}$ for the three events analyzed in this paper are shown. The shadowed area in Figure 14 (top and middle) corresponds to the periods when a significant energy release from the solar wind into the terrestrial magnetosphere takes place. As the aim is to forecast the response of this energy release in the terrestrial environment, the shadowed areas in Figure 14 (bottom) have been shifted about $1 \mathrm{~h}$ in order to take into account the delay from the L1 point to the Earth, and the magnetospheric response time [Gonzalez and Echer, 2005]. The periods of large energy release are well correlated to the periods of decrease in Dst*. From Figure 14 it is easy to conclude that the more negative $Q$ values are, the more negative $D s t^{*}$ index is, as expected. However, to be conclusive in the comparison of the geoeffectiveness of different events, this comparison should be done taking into account the total energy released to the magnetosphere in each geomagnetic storm, and not using values of the injection function at a given time.

[33] Considering only the source term in equation (2), the variation in $D s t^{*}$ from quiet time up to its minimum value can be related to the total energy released at a given storm as follows:

$$
\Delta D s t^{*}=\int_{t_{1}}^{t_{2}} Q(t) d t
$$

The interval $\left(t_{1}, t_{2}\right)$ corresponds to the time interval when the injection function is not zero, that is, when the energy is being transferred from the solar wind to the magnetosphere (main phase of the storm). It should be noted, that the same energy can be injected either with large values of $Q$ during short periods of time or with less intense $Q$ values lasting longer periods of time. Nevertheless, the decrease in $D s t^{*}$ is faster in the former case. From the point of view of predictions, this is an important factor since a sudden decrease in $D s t^{*}$ can lead to the generation of induced currents.

[34] Following equation (5), we have compared the geoeffectiveness of the three events analyzed in this paper, as measured from $\Delta D s t^{*}$, and the total energy released, as computed from the temporal integral of $Q(t)$ extended to the shadowed zones in Figure 14. Figure 15 shows the measured variation of $D s t^{*}$ versus the total energy released. It can be seen from Figure 15 that the relative geoeffectiveness of the events is well explained on the base of the L1 

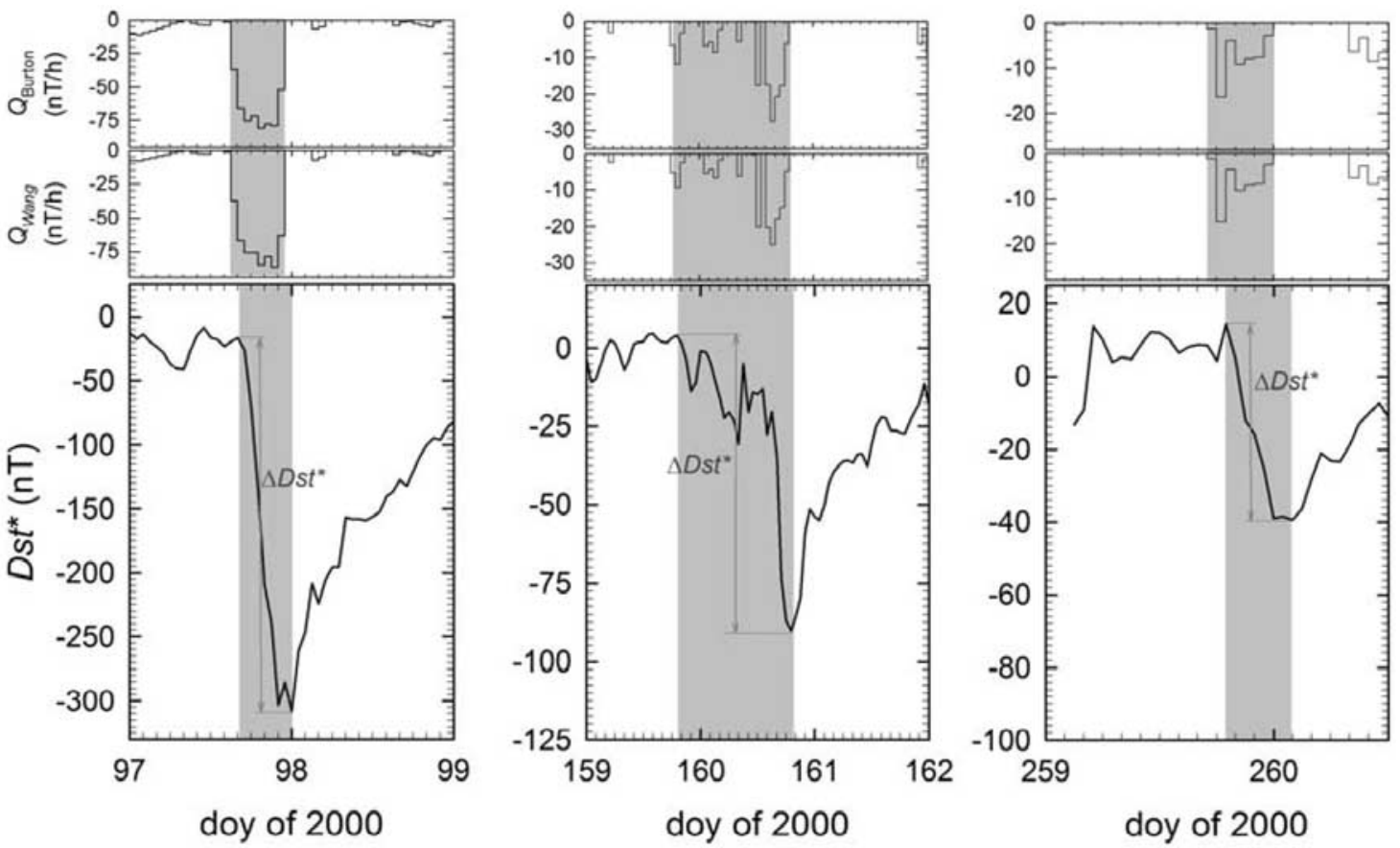

Figure 14. (top and middle) The Burton and Wang injection functions for the three events analyzed and (bottom) pressure-corrected Dst (Dst*). See text for details about shadowed zones.

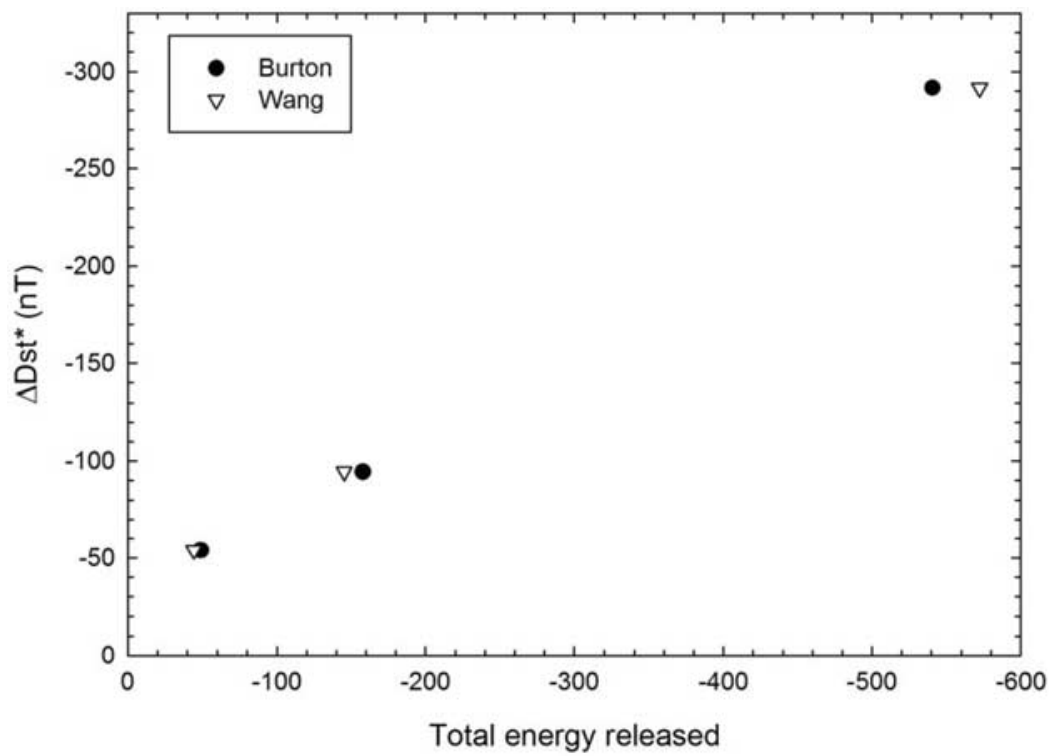

Figure 15. Experimental $\Delta D s t^{*}$ versus total energy released for the three events analyzed in this paper. $\Delta D s t^{*}$ corresponds to the difference between the minimum and quiet time values of measured $D s t^{*}$ for each event (see Figure 14 for details). The total energy released has been computed using equation (5), being $Q(t)$ expressed in nT/hour and the time interval $\left(t_{1}, t_{2}\right)$ corresponds to the shadowed area of Figure 14 for each event. 
measurements. In fact, a linear regression analysis provides very accurate results, with $r^{2}$ over 0.9 for both injection functions.

\section{Discussion and Conclusions}

[35] We have studied three fast frontside full halo CMEs that showed similar on-disk signatures. Nevertheless, their geomagnetic responses were very different. The location of the source region on the Sun was a factor producing an effect opposite to what can be expected, meaning that the two close to central meridian events were less geoeffective than the one with the source region close to the limb. We have tried to establish what factors (that can be deduced from available solar observations) may be useful for the ultimate goal to predict the strength and duration of the southward IMF component.

[36] The solar source region of the fast $(1550 \mathrm{~km} / \mathrm{s})$ full halo CME on 12 September 2000 was situated close to disk center (S17W09). However, only its associated shock arrived to the Earth because of the fact that the halo CME was highly asymmetric with its bulk going south. There was no strong negative $B_{z}$ associated with the postshock solar wind flow resulting in a weak geomagnetic disturbance (minimum Dst reached $-39 \mathrm{nT}$ ). The CME did not seem to interact with any other structure in its cruise to the Earth. Our result confirms the study by Moon et al. [2005] who concluded that the mass distribution in the CME correlates well with the CME geoeffectiveness. We note, however, that Moon et al. [2005] did not investigate the interplanetary data and thus could not reliably separate the effects of the CME nonarrival and arrival of a nongeoeffective CME.

[37] The solar source region of the fast $(1119 \mathrm{~km} / \mathrm{s})$ full halo CME on 6 June 2000 was situated close to disk center (N20E18). A shock followed by an ICME arrived to the Earth. Even though $B_{z}$ in the postshock sheath region reached $-20 \mathrm{nT}$, the duration of this enhancement was too short, resulting only in a moderate geomagnetic storm (minimum Dst reached $-90 \mathrm{nT}$ ). In this case the Earth has passed through a leg of the magnetic cloud. Such geometry of encounter is not favorable for the appearance of strong long-lasting southward $B_{z}$. There was a CME ejected from the Sun about $25 \mathrm{~min}$ before the halo CME, so an interaction between both CMEs was possible and some imprints of the first CME could possibly be detected in situ.

[38] The solar source region of the fast $(1188 \mathrm{~km} / \mathrm{s})$ full halo CME on 4 April 2000 was situated close to the west limb (N16W66). A strong shock arrived to the Earth, followed by a sheath with strong and long-lasting southward $B_{z}$, producing a superintense geomagnetic storm (minimum Dst reached -288 nT). We argue that this event was the result of a strong and fast CME overtaking a previous slower one. This might have contributed to the strong charge states seen in the sheath region and to the orientation of the CME driven shock, eventually contributing also to the high geoeffectiveness that resulted when it arrived at the magnetosphere. Another possibility is the strong compression at the eastern flank of the sheath and eastward deflection of the fast ICME by the magnetic tension force from the IMF in the ambient slower solar wind flow. We also note that the CME brightness asymmetry (the factor that determined a weak geoeffectiveness of the 12 September CME) does not suggest a weak geoeffectiveness in this case, contrary to the suggestion by Moon et al. [2005].

[39] Even the north-south CME brightness asymmetry may be not an unambiguous factor in determination of the CME direction. Several cases of a significant brightness asymmetry in CMEs, which nevertheless arrived to the Earth, were reported, for example the 12 May 1997 CME [Plunkett et al., 1998; Webb et al., 2000] and the 18 November 2003 CME [Gopalswamy et al., 2005]. However, both the 12 May 1997 and 18 November 2003 CMEs occurred either in the rising or in the declining phase of the solar cycle, when the polar coronal holes were well developed. This may have led to the deflection of the CME toward the equator, in a process specified, e.g., by Cremades and Bothmer [2004]. On the contrary, the 12 September 2000 CME occurred near the peak of the solar activity cycle. There were no polar coronal holes at all and the CME thus propagated unimpeded.

[40] We note that the associated flare magnitude was not a good indicator of the resulting event geoeffectiveness, in agreement with previous studies [e.g., Gosling, 1993; Zhang et al., 2007]. Indeed, the C9.7 flare on 4 April 2000 was associated with a strongly geoeffective CME, whereas the X2.3 flare on 6 June 2000 was associated only with a moderately geoeffective CME. Another frequently cited geoeffective parameter, the observed CME speed [e.g., Yurchyshyn et al., 2004], would not have helped either in predicting the geoeffectiveness as all three CMEs were fast (with speeds higher than $1100 \mathrm{~km} / \mathrm{s}$ ).

[41] None of the described events was associated with a well-defined magnetic cloud crossed close to its apex, even for the events with the source region close to the central meridian. This indicates that attempts to associate the magnetic cloud axis orientations with solar signatures (e.g., filament or posteruptive arcade orientations [see, e.g., Marubashi, 1986, 1997; Bothmer and Schwenn, 1997; Yurchyshyn et al., 2001]) may not be sufficient in certain exceptional events (as the ones presented here) to predict the IMF configuration. Our results demonstrate that for this kind of events, even if erupting filaments and posteruptive arcades are observed, it may be difficult to determine on the base of solar observations if a magnetic cloud would be detected at the Earth at all.

[42] The analysis of the in situ measurements provide us with a way to explain the relative geoeffectiveness of the three events analyzed: a bigger temporal integral of $Q(t)$ is related to a larger energy input from the solar wind into the magnetosphere and thus to a more geoeffective event. In this manner, the L1-Earth link in the Sun-Earth con- 
nection chain of events is much better understood than the previous one, the Sun-L1 phase.

[43] Meaningful statistical conclusions can be obtained from the study of many events in which a relation between solar eruptions and their magnetospheric consequences can be established. Nevertheless, there exist exceptional events that do not follow general trends. The clues needed to understand their geoeffectiveness on the base of solar observations remain difficult to interpret. For each event, we mentioned several possible factors that can be important for the halo CME geoeffectiveness. One of the factors determining the final geoeffectiveness of CMEs can be the interaction with other CMEs or with fast solar wind streams. Note that out of the three cases listed in this work, the case in which the interaction is clearer in the data (the 4-6 April event) is the one that originally had the smallest chance to create a significant geomagnetic storm (because of the source region position close to the limb) but nevertheless produced the strongest one. On the other hand, the event that seemingly interacted less with other structures (the 12 September CME) produced a weak geomagnetic storm, even though its source region was situated close to the solar central meridian (potentially geoeffective position). Cid et al. [2008] found that 5 out of 11 superintense geomagnetic storms were the result of interaction between different ICMEs. The result of this kind of interaction is difficult to predict and at the moment there is no efficient way of providing reliable forecasts in these cases. One possible technique that might help improving our predictions is the use of data from both STEREO A and B spacecraft in order to better determine the direction of propagation of CMEs [e.g., Mierla et al., 2008]. A second valuable contribution may come from placing in situ observatories closer to the Sun, such as Solar Orbiter, increasing in this way the alert time before the disturbances arrive at the Earth.

[44] Last, we would like to mention that our results stress the difficulties related to making accurate space weather forecasts with the current knowledge and tools at hand. Our interpretations are provided a posteriori and may not be always possible in real-time. We, however, stress the additional factors (e.g., mass distribution within the CME, passage through a leg of the magnetic cloud, strong magnetic fields in the sheath region) that have to be taken into account when making real-time predictions. A forecaster may react differently to the data if he takes this information about an event into account. The influence that improvements in the field would have for various practical purposes (e.g., for satellite operations, radio communications, global positioning system applications, power grid and pipeline maintenance, etc.) is unquestionable. All these technological applications depend on the state of the magnetosphere that may be disturbed by the arrival of ICMEs. Predicting potential geoeffectiveness of CMEs on the base of solar observations is thus an ultimate goal of the space weather science, and our work is a step in this direction.
[45] Acknowledgments. The present study was developed in the frame of the "International Teams" program from the International Space Science Institute (ISSI) in Bern, Switzerland, the authors thank this institution for the great support provided during the course of this work in the framework of "The stages of the Sun-Earth connection" project. L. Rodriguez and A. N. Zhukov acknowledge support from the Belgian Federal Science Policy Office through the ESAPRODEX program. C. Cid, Y. Cerrato, and E. Saiz acknowledge financial support from Comisión Interministerial de Ciencia y Tecnología (CICYT) of Spain (ESP 2005-07290-C0201 and ESP 2006-08459). C. H. Mandrini and S. Dasso thank grants UBACyT X329, PIP 6220 (CONICET), and PICTs 03-12187 and 03-14163 (ANPCyT). S. Dasso thanks the Argentinean grant UBACyT X425 and PICT 03-33370 (ANPCyT). C. H. Mandrini, H. Cremades, and S. Dasso are members of the Carrera del Investigador Científico, CONICET. A. Aran acknowledges the financial support of the Spanish Ministerio de Educación y Ciencia under the project AYA2004-03022. We are grateful to the ACE/SWEPAM, ACE/ MAG, and ACE/SWICS teams for the data used for this work. The CME catalog is generated and maintained at the CDAW Data Center by NASA and the Catholic University of America in cooperation with the Naval Research Laboratory. EIT, LASCO, and MDI data are a courtesy of SOHO/EIT, SOHO/LASCO, and SOHO/MDI consortiums, respectively. SOHO is a project of international cooperation between ESA and NASA. The $H \alpha$ images employed in this work were provided by the Big Bear Solar Observatory/New Jersey Institute of Technology. We would like to thank the WDCKyoto for providing the Dst index.

\section{References}

Akasofu, S.-I., and C. F. Fry (1986), A first generation numerical geomagnetic storm prediction scheme, Planet. Space Sci., 34(1), 77-92, doi:10.1016/0032-0633(86)90105-4.

Arnoldy, R. L. (1971), Signature in the interplanetary medium for substorms, J. Geophys. Res., 76(22), 5189-5201, doi:10.1029/ JA076i022p05189.

Bothmer, V., and I. A. Daglis (Eds.) (2007), Space Weather---Physics and Effects, Springer, Chichester, U. K.

Bothmer, V., and R. Schwenn (1997), The structure and origin of magnetic clouds in the solar wind, Ann. Geophys., 16(1), 1-24, doi:10.1007/s00585-997-0001-x.

Bothmer, V., and A. Zhukov (2007), The Sun as the prime source of space weather, in Space Weather---Physics and Effects, edited by V. Bothmer and I. A. Daglis, pp. $31-102$, Springer, Chichester, U. K. Brueckner, G. E., et al. (1995), The Large Angle Spectroscopic Coronagraph (LASCO), Sol. Phys., 162, 357-402, doi:10.1007/BF00733434.

Burlaga, L. F. E. (1991), Magnetic clouds, in Physics of the Inner Heliosphere II, edited by R. Schwenn and E. Marsch, pp. 1-22, Springer, Berlin.

Burton, R. K., R. L. McPherron, and C. T. Russell (1975), An empirical relationship between interplanetary conditions and Dst, J. Geophys. Res., 80(31), 4204, doi:10.1029/JA080i031p04204.

Cid, C., E. Saiz, and Y. Cerrato (2008), Comment on "Interplanetary conditions leading to superintense geomagnetic storms (Dst $\leq$ $-250 \mathrm{nT}$ ) during solar cycle 23" by E. Echer et al., Geophys. Res. Lett., 35, L21107, doi:10.1029/2008GL034731.

Cremades, H., and V. Bothmer (2004), On the three-dimensional configuration of coronal mass ejections, Astron. Astrophys., 422, 307-322. 
Dasso, S., D. Gómez, and C. H. Mandrini (2002), Ring current decay rates of magnetic storms: A statistical study from 1957 to 1998, J. Geophys. Res., 107(A5), 1059, doi:10.1029/2000JA000430.

Dasso, S., C. H. Mandrini, P. Dèmoulin, and M. L. Luoni (2006), A new model-independent method to compute magnetic helicity in magnetic clouds, Astron. Astrophys., 455, 349-359.

Delaboudinière, J.-P., et al. (1995), EIT: Extreme-ultraviolet Imaging Telescope for the SOHO mission, Sol. Phys., 162(1-2), 291-312, doi:10.1007/BF00733432.

Delannée, C., J.-P. Delaboudiniére, and P. Lamy (2000), Observation of the origin of CMEs in the low corona, Astron. Astrophys., 355, $725-742$.

Dessler, A. J., and E. N. Parker (1959), Hydromagnetic theory of geomagnetic storms, J. Geophys. Res., 64(12), 2239-2252, doi:10.1029/ JZ064i012p02239.

Dungey, J. W. (1961), The steady state of the Chapman-Ferraro problem in two dimensions, J. Geophys. Res., 66(4), 1043-1047, doi:10.1029/JZ066i004p01043.

Galvin, A. B., F. M. Ipavich, G. Gloeckler, D. Hovestadt, and B. T. Tsurutani (1987), Solar wind iron charge states preceding a driver plasma, J. Geophys. Res., 92, 12,069--12,081, doi:10.1029/ JA092iA11p12069.

Gonzalez, W. D., and E. Echer (2005), A study on the peak Dst and peak negative $\mathrm{Bz}$ relationship during intense geomagnetic storms, Geophys. Res. Lett., 32, L18103, doi:10.1029/2005GL023486.

Gonzalez, W. D., J. A. Joselyn, Y. Kamide, H. W. Kroehl, G. Rostoker, B. T. Tsurutani, and V. M. Vasyliunas (1994), What is a geomagnetic storm?, J. Geophys. Res., 99(A4), 5771-5792, doi:10.1029/93JA02867.

Gonzalez, W. D., B. T. Tsurutani, P. McIntosh, and A. L. Clúa de Gonzalez (1996), Coronal Hole-Active Region-Current Sheet (CHARCS) association with intense interplanetary and geomagnetic activity, Geophys. Res. Lett., 23(19), 2577-2580.

Gonzalez, W. D., B. T. Tsurutani, and A. L. Clúa de Gonzalez (1999), Interplanetary origin of geomagnetic storms, Space Sci. Rev., 88(3/4), 529-562, doi:10.1023/A:1005160129098.

Gopalswamy, N. (2002), Relation between coronal mass ejections and their interplanetary counterparts, in Proceedings of the COSPAR Colloquium, Beijing, China, vol. 14, edited by H. Wang and R. Xu, p. 157, Pergamon, Boston, Mass.

Gopalswamy, N., S. Yashiro, G. Michalek, H. Xie, R. P. Lepping, and R. A. Howard (2005), Solar source of the largest geomagnetic storm of cycle 23, Geophys. Res. Lett., 32, L12S09, doi:10.1029/2004GL021639.

Gopalswamy, N., S. Yashiro, and S. Akiyama (2007), Geoeffectiveness of halo coronal mass ejections, J. Geophys. Res., 112, A06112, doi:10.1029/2006JA012149.

Gosling, J. T. (1993), The solar flare myth, J. Geophys. Res., 98(A11), 18,937-18,949, doi:10.1029/93JA01896.

Gosling, J. T., and D. J. McComas (1987), Field line draping about a fast coronal mass ejecta: A source of strong out-of-ecliptic interplanetary magnetic field, Geophys. Res. Lett., 14(4), 355-358, doi:10.1029/ GL014i004p00355.

Gosling, J. T., M. F. Thomsen, S. J. Bame, and R. D. Zwickl (1987), The eastward deflection of fast coronal mass ejecta in interplanetary space, J. Geophys. Res., 92(A11), 12,399-12,406, doi:10.1029/ JA092iA11p12399.

Gosling, J. T., S. J. Bame, D. J. McComas, and J. L. Phillips (1990), Coronal mass ejections and large geomagnetic storms, Geophys. Res. Lett., 17, 901-904, doi:10.1029/GL017i007p00901.

Hidalgo, M. A., T. Nieves-Chinchilla, and C. Cid (2002), Elliptical crosssection model for the magnetic topology of magnetic clouds, Geophys. Res. Lett., 29(13), 1637, doi:10.1029/2001GL013875.

Hirshberg, J., and D. S. Colburn (1969), Interplanetary field and geomagnetic variations: A unified view, Planet. Space Sci., 17, 1183-1206, doi:10.1016/0032-0633(69)90010-5.

Howard, R. A., D. J. Michels, N. R. Sheeley Jr., and M. J. Koomen (1982), The observation of a coronal transient directed at earth, Astrophys. J., 263, L101-L104.

Huttunen, K. E. J., H. E. J. Koskinen, T. I. Pulkkinen, A. Pulkkinen, M. Palmroth, E. G. D. Reeves, and H. J. Singer (2002), April 2000 magnetic storm: Solar wind driver and magnetospheric response, J. Geophys. Res., 107(A12), 1440, doi:10.1029/2001JA009154.

Jadav, R. M., K. N. Iyer, H. P. Joshi, and H. O. Vats (2005), Coronal mass ejection of 4 April 2000 and associated space weather effects, Planet. Space Sci., 53(6), 671-679, doi:10.1016/j.pss.2005.01.002.

Manoharan, P. K., et al. (2004), Influence of coronal mass ejection interaction on propagation of interplanetary shocks, J. Geophys. Res., 109, A06109, doi:10.1029/2003JA010300.

Martin, S. F. (2003), Signs of helicity in solar prominences and related features, Adv. Space Res., 32(10), 1883-1893, doi:10.1016/S02731177(03)90622-3.

Marubashi, K. (1986), Structure of the interplanetary magnetic clouds and their solar origins, Adv. Space Res., 6, 335-338, doi:10.1016/02731177(86)90172-9.

Marubashi, K. (1997), Interplanetary magnetic flux ropes and solar flaments, in Coronal Mass Ejections, Geophys. Monograph. Ser., vol. 99, edited by N. Crooker, J. Joselyn, and J. Feynman, pp. $147-$ 156, AGU, Washington, D. C.

Meng, C.-I., B. Tsurutani, K. Kawasaki, and S.-I. Akasofu (1973), Crosscorrelation analysis of the $\mathrm{AE}$ index and the interplanetary magnetic field $B_{z}$ component, J. Geophys. Res., 73(4), 617-629, doi:10.1029/JA078i004p00617.

Menvielle, M., and A. Berthelier (1991), The K-derived planetary indices: Description and availability, Rev. Geophys., 29, 415-432, doi:10.1029/91RG00994.

Mierla, M., J. Davila, W. Thompson, B. Inhester, N. Srivastava, M. Kramar, O. C. St. Cyr, G. Stenborg, and R. A. Howard (2008), Quick method for estimating the propagation direction of coronal mass ejections using STEREO-COR1 images, Sol. Phys., 252(2), 385-396, doi:10.1007/s11207-008-9267-8.

Moon, Y.-J., K.-S. Cho, M. Dryer, Y.-H. Kim, S.-C. Bong, J. Chae, and Y. D. Park (2005), New geoeffective parameters of very fast halo coronal mass ejections, Astrophys. J., 624(1), 414-419.

O'Brien, T. P., and R. L. McPherron (2000), An empirical phase-space analysis of ring current dynamics: Solar wind control of injection and decay, J. Geophys. Res., 105, $7707-$-7719, doi:10.1029/ 1998JA000437.

Patel, V. L., and M. J. Wiskerchen (1975), Interplanetary field and plasma during initial phase of geomagnetic storms, J. Geomag. Geoelectr., 27, 363-382.

Perreault, P., and S.-I. Akasofu (1978), A study of geomagnetic storms, Geophys. J. Int., 54(3), 547-573, doi:10.1111/j.1365-246X.1978. tb05494.x.

Piddington, J. H. (1963), Theories of the geomagnetic storm main phase, Planet. Space Sci., 11, 1277-1288, doi:10.1016/0032-0633 (63) $90232-0$.

Plunkett, S. P., B. J. Thompson, R. A. Howard, D. J. Michels, O. C. St. Cyr, S. J. Tappin, R. Schwenn, and P. L. Lamy (1998), LASCO observations of an Earth-directed coronal mass ejection on May 12, 1997, Geophys. Res. Lett., 25(14), 2477-2480, doi:10.1029/98GL50307.

Richardson, I. G., and H. V. Cane (2004), Identification of interplanetary coronal mass ejections at $1 \mathrm{AU}$ using multiple solar wind plasma composition anomalies, J. Geophys. Res., 109, A09104, doi:10.1029/ 2004JA010598.

Rostoker, G., and C.-G. Fälthammar (1967), Relationship between changes in the interplanetary magnetic field and variations in the magnetic field at the Earth's surface, J. Geophys. Res., 72(23), $5853-$ 5863, doi:10.1029/JZ072i023p05853.

Russell, C. T., R. L. McPherron, and R. K. Burton (1974), On the cause of geomagnetic storms, J. Geophys. Res., 79, 1105-1109, doi:10.1029/ JA079i007p01105.

Schwenn, R. (2006), Space weather: The solar perspective, Living Rev. Solar Phys., 3, 2.

Schwenn, R., A. Dal Lago, E. Huttunen, and W. D. Gonzalez (2005), The association of coronal mass ejections with their effects near the Earth, Ann. Geophys., 23, 1033-1059.

Sckopke, N. (1966), A general relation between the energy of trapped particles and the disturbance field near the Earth, J. Geophys. Res., $71,3125-3130$. 
Siscoe, G., P. J. MacNeice, and D. Odstrcil (2007), East-west asymmetry in coronal mass ejection geoeffectiveness, Space Weather, 5, S04002, doi:10.1029/2006SW000286.

Sonnerup, B. U. Õ., and L. J. Cahill (1967), Magnetopause structure and attitude from Explorer 12 observations, J. Geophys. Res., 72, 171-183, doi:10.1029/JZ072i001p00171.

St. Cyr, O. C., et al. (2000), Properties of coronal mass ejections: SOHO LASCO observations from January 1996 to June 1998, J. Geophys. Res., 105, 18,169-18,185, doi:10.1029/1999JA000381.

Sugiura, M. (1965), Hourly values of equatorial Dst for the IGY, Ann. Int. Geophys. Year, 35, 9-45.

Sugiura, M., and T. Kamei (1991), Equatoriel Dst index, 1957-1986, Int. Assoc. Geomagn. Aeron. Bull., 40, 246.

Thompson, B. J., E. W. Cliver, N. Nitta, C. Delannée, and J.-P. Delaboudinière (2000), Coronal dimmings and energetic CMEs in April-- May 1998, Geophys. Res. Lett., 27(10), 1431 -- 1434, doi:10.1029/1999GL003668.

Tsurutani, B. T., E. J. Smith, W. D. Gonzalez, F. Tang, and S.-I. Akasofu (1988), Origin of interplanetary southward magnetic fields responsible for major magnetic storms near solar maximum (1978-1979), J. Geophys. Res., 93, 8519-8531, doi:10.1029/JA093iA08p08519.

Wang, C. B., J. K. Chao, and C. H. Lin (2003), Influence of the solar wind dynamic pressure on the decay and injection of the ring current, J. Geophys. Res., 108(A9), 1341, doi:10.1029/2003JA009851.

Wang, Y. M., P. Z. Ye, S. Wang, and X. H. Xue (2003), An interplanetary cause of large geomagnetic storms: Fast forward shock overtaking preceding magnetic cloud, Geophys. Res. Lett., 30(13), 1700, doi:10.1029/2002GL016861.

Webb, D. F., R. P. Lepping, L. F. Burlaga, C. E. DeForest, D. E. Larson, S. F. Martin, S. P. Plunkett, and D. M. Rust (2000), The origin and development of the May 1997 magnetic cloud, J. Geophys. Res., 105(A12), 27,251-27,260, doi:10.1029/2000JA000021.

Xie, H., N. Gopalswamy, P. K. Manoharan, A. Lara, S. Yashiro, and S. Lepri (2006), Long-lived geomagnetic storms and coronal mass ejections, J. Geophys. Res., 111, A01103, doi:10.1029/2005JA011287.

Yashiro, S., N. Gopalswamy, G. Michalek, O. C. St. Cyr, S. P. Plunkett, N. B. Rich, and R. A. Howard (2004), A catalog of white light coronal mass ejections observed by the SOHO spacecraft, J. Geophys. Res., 109, A07105, doi:10.1029/2003JA010282.

Yurchyshyn, V. B., H. Wang, P. R. Good, and Y. Deng (2001), Orientation of the magnetic fields in interplanetary flux ropes and solar filaments, Astrophys. J., 563, 381-388, doi:10.1086/323778.
Yurchyshyn, V. B., H. Wang, and V. Abramenko (2004), Correlation between speeds of coronal mass ejections and the intensity of geomagnetic storms, Space Weather, 2, S02001, doi:10.1029/ 2003SW000020.

Zhang, J., et al. (2007), Solar and interplanetary sources of major geomagnetic storms (Dst $<=-100 \mathrm{nT}$ ) during 1996-2005, J. Geophys. Res., 112, A10102, doi:10.1029/2007JA012321.

Zhukov, A. N. (2005), Solar sources of geoeffective CMEs: A SOHO/ EIT view, in Coronal and Stellar Mass Ejections, IAU Symposium Proceedings of the International Astronomical Union 226, edited by K. Dere, J. Wang, and Y. Yan, pp. 437-447, Cambridge Univ. Press, Cambridge, U. K.

Zhukov, A. N. (2007), Using CME observations for geomagnetic storm forecasting, in Space Weather: Research Towards Applications in Europe 2nd European Space Weather Week (ESWW2), vol. 344, edited by J. Lilensten, pp. 5-13, Eur. Space Agency, Noordwijk, Netherlands. Zhukov, A. N., and I. S. Veselovsky (2007), Global coronal mass ejections, Astrophys. J., 664, L131 - L143, doi:10.1086/520928.

Zurbuchen, T. H., and I. Richardson (2006), In-situ solar wind and field signatures of interplanetary coronal mass ejections, Space Sci. Rev., 123(1-3), 31-43, doi:10.1007/s11214-006-9010-4

A. Aran, Department of Astronomy and Meteorology, University of Barcelona, Marti i Franques 1, 7th floor, E-08028 Barcelona, Spain.

Y. Cerrato, C. Cid, and E. Saiz, Department of Physics, University of Alcala, Ctra. Nacional II, Km. 33,600, E-28871 Alcala de Henares, Spain.

H. Cremades, Facultad Regional Mendoza, Universidad Tecnológica Nacional, Rodriguez 273, 5502 Mendoza, Argentina.

S. Dasso and C. Mandrini, Instituto de Astronomía y Física de Espacio, CONICET, Universidad de Buenos Aires, 1428 Buenos Aires, Argentina.

M. Menvielle, Centre d'Etude des Environnements Terrestre et Planétaires, 10-12 Avenue de l'Europe, F-78140 Vélizy-Villacoublay, France.

S. Poedts, Centre for Plasma Astrophysics, Catholic University Leuven, Celestijnenlaan 200 B, B-3001 Leuven, Belgium.

L. Rodriguez and A. N. Zhukov, Solar-Terrestrial Center of Excellence, SIDC, Royal Observatory of Belgium, Avenue Circulaire 3, B-1180 Brussels, Belgium. (rodriguez@oma.be)

B. Schmieder, Observatoire de Paris, 5 Place Jules Janssen, F-92195 Meudon, France. 